\title{
TORSIONAL AND BENDING VIBRATION MEASUREMENT ON ROTORS USING LASER TECHNOLOGY
}

\author{
T.J. MILES*, M. LUCAS**, N.A. HALLIWELL and S.J. ROTHBERG \\ Department of Mechanical Engineering, Loughborough University, UK. \\ *Now at Rolls-Royce Strategic Research Centre, Derby, UK \\ **Now at Department of Mechanical Engineering, University of Glasgow, UK.
}

Total Number of Main Text Pages: 30

Total Number of Figures: 19 in 26 pieces

Short Title: TORSIONAL AND BENDING VIBRATION MEASUREMENT

Address for Correspondence:

Dr S.J. Rothberg

Department of Mechanical Engineering

Loughborough University

LOUGHBOROUGH

Leicestershire LE11 3TU. 


\section{SUMMARY}

Based on the principles of laser Doppler velocimetry, the laser torsional vibrometer (LTV) was developed for non-contact measurement of torsional oscillation of rotating shafts, offering significant advantages over conventional techniques. This paper describes comprehensive theory to account for the sensitivity of the LTV's measurements to shaft motion in all degrees of freedom. The optical geometry of the LTV offers inherent immunity to translational motion of the target shaft, either axial or radial. However, its measurements are sensitive to angular lateral vibration of the shaft. The significance of this sensitivity is compared with the instrument noise floor and typical torsional and lateral vibration levels. Optimum alignments of the instrument are then specified to ensure effective immunity to all lateral motion in typical applications. To overcome this problem more reliably, a new technique is proposed permitting unambiguous measurement of pure torsional vibration in situations where use of a single LTV demonstrates unacceptable sensitivity to angular lateral vibrations. Practical application of this technology is demonstrated with torsional vibration measurements from a diesel engine crankshaft. Simultaneously, previously unattained measurements of shaft bending vibration measurements are made. The first bending mode of the crankshaft was identified and its vibration amplitude and damping estimated. This application of laser vibrometry for non-contact measurements of shaft vibration represents a further step forward in the use of this technology for machinery diagnostics. 


\section{INTRODUCTION}

Torsional and bending vibrations are both important in rotating shaft systems. Typical torsional vibration problems in automotive, marine propulsion and industrial applications include oscillation of crankshafts, drive-shafts, and component wear in gearboxes. In these cases shaft cracking, coupling deterioration and gear failure can result $[1,2]$. A number of situations can give rise to shaft bending (or, more generally, angular lateral) vibrations, including unbalance, misalignment, bearing faults, rubs and the effects of gears, blades and vanes [3]. Bending vibration of reciprocating engine crankshafts is particularly important in automotive NVH studies. Low order bending vibrations are of concern in engine reliability and high order vibrations are influential on power-plant noise and vibration [4]. Crankshaft bending vibration is also believed to be the main source of engine 'rumble', a troublesome low and mid-frequency noise that reduces passenger comfort, particularly during acceleration [5]. The difficulty of obtaining measurements directly from rotating components has until recently prevented routine experimental analysis of these quantities.

The measurement of translational vibration velocity (on non-rotating structures) with laser Doppler velocimetry (LDV) [6] is now a well-established experimental technique. The basic principle of LDV requires the detection of the Doppler frequency shift in the coherent light scattered from a moving object. The magnitude of this Doppler shift is proportional to the instantaneous target velocity in the direction of incidence of the laser beam. The laser torsional vibrometer (LTV) [7-9] extended the application of this technology, achieving accurate non-contact measurement of the torsional vibration of rotating components and overcoming the limitations of conventional instrumentation [10]. It has seen use in a range of 
applications, from examining speed fluctuations of tape recorder drives to failure diagnosis of torsional dampers on large diesel engines.

The ingeniously simple optical arrangement provides a compact and robust solution through an arrangement of two parallel laser beams. The light from a single laser is divided into two beams which impinge on the shaft and are Doppler shifted in proportion to the surface velocity at their respective points of incidence. When this backscattered light is recombined and mixed on a photodetector, a beat is produced in the photodetector output at the frequency difference between each Doppler shifted beam. This beat is proportional to target rotation speed, $\omega_{R}$, as follows [9]:

$$
f_{\text {beat }}=\left|\left(\frac{2 \mu d}{\lambda}\right) \omega_{R} \cos \theta\right|
$$

where $\mu$ is the refractive index of the surrounding medium (unity for air), $d$ is the laser beam separation, $\lambda$ is the laser wavelength and $\theta$ is the incidence angle between the normal to the laser beam plane and rotational axis of the target shaft. The photodetector output is analysed by a suitable Doppler signal processor, essentially a frequency to voltage converter, which produces a time-resolved analogue of the beat frequency. Torsional vibration is seen as a fluctuation in rotation speed of the shaft element of interest, with a corresponding fluctuation in beat frequency and hence instrument output voltage.

Measurements with the LTV are unaffected by the shape of the target, allowing use on components of arbitrary cross-section. Use on the side of a shaft or on the end-face is possible and this is advantageous where access is restricted. Additionally, the system uses a 
minimum of optical components and has straightforward signal processing requirements. Often retro-reflective tape is applied to the target shaft to facilitate use of a low powered laser. The LTV has been demonstrated to give good agreement with existing methods of measuring torsional vibration [9] and its optical arrangement has been adopted in a commercial instrument [11].

The Doppler shift of a single laser beam incident on a rotating shaft can be related to the motion of the target in its six degrees of freedom. The unique optical geometry of the LTV offers immunity to shaft axial or radial motion, such as the cylindrical whirl orbit of Figure 1a, as well as equivalent motion of the instrument itself. This paper examines the instrument's measurable sensitivity to angular vibration, an issue which has not been explicitly addressed in previous work. During such motion, the shaft rotation vector undergoes a change of direction, effectively changing the incidence angle, $\theta$, and influencing the measurements. A simple example of this is the conical whirl orbit of Figure 1b. This motion will subsequently be referred to as angular lateral vibration which also includes whirling of a flexible shaft. In later discussion of crankshaft motion, this is more specifically described as bending vibration.

Angular lateral vibration of a rotating shaft is inherently difficult to measure and, as with torsional vibration, measurement of actual shaft motion must be obtained directly from the component, often with complicated instrumentation requirements. Previously reported measurements have used accelerometers attached to a stationary housing which is carried by a retro-fitted bearing on the shaft of interest $[12,13]$. Studies of engine crankshaft vibration have also included measurements of shaft lateral motion by eddy current displacement sensors [14] and strain gauges attached to the shaft [15]. In an additional study, closely 
related to this one, angular vibration measurements have also been taken from a non-rotating structure using a novel laser Doppler transducer [16].

Comprehensive theory is presented in this paper to consider, for the first time, the effects of shaft motion in all degrees of freedom on torsional vibration measurements obtained with an LTV. Subsequent discussion, based on experimental results, quantifies the sensitivity of the instrument to angular lateral vibration highlighting when the potential for measurement ambiguity exists. In the context of the instrument noise floor and typical torsional and angular lateral vibration levels found in practice, guidelines are then proposed for accurate assessment of genuine torsional oscillation by optimisation of the optical geometry used. In this way complete immunity to all lateral shaft vibrations can be achieved.

Ultimately, a solution is proposed which permits unambiguous measurement of shaft torsional vibration in situations where use of a single LTV would otherwise show unacceptable sensitivity to angular lateral motion. Measurements from an engine crankshaft demonstrate the practical application of this new technique. Simultaneously, this technique can provide a measurement of the bending vibration of a rotating shaft and experimental results are presented which give an insight into the complex lateral motion of a reciprocating engine crankshaft.

\section{ADVANCES IN THE THEORY OF OPERATION OF THE LTV}

\subsection{GENERAL DEFINITION OF MOTION OF A POINT ON A ROTATING SHAFT}

Consider the general case of a point $P$ on the surface of an arbitrarily shaped shaft, located by

position vector $\overrightarrow{\boldsymbol{r}_{\boldsymbol{p}}}$ with respect to the co-ordinate axes, as illustrated in Figure 2. The main 
co-ordinate system OXYZ has its origin fixed on the undeflected shaft rotation axis (i.e. in the absence of any vibration) which also defines $\hat{\mathbf{z}}$. The $X$-, $Y$ - and $Z$-axes maintain their directions during vibration.

The instantaneous velocity vector can be related to motion in six degrees-of-freedom, comprising the three translational motions $\overrightarrow{V_{x}}, \overrightarrow{V_{y}}$ and $\overrightarrow{V_{z}}$ and components related to the angular motion of the shaft. These are considered as the shaft rotation at angular velocity $\omega_{R}$ about an axis defined by the vector $\hat{\mathbf{z}}_{\boldsymbol{R}}$ and angular lateral motion which results in the angular displacement of $\hat{\mathbf{z}}_{\boldsymbol{R}}$ relative to $\hat{\mathbf{z}}$. This latter motion is described by finite rotations about the two co-ordinate axes, $X$ and $Y$, making use of the unit vectors $\hat{\boldsymbol{x}}$ and $\hat{\boldsymbol{y}}$. These rotations of the shaft axis are conventionally described as pitch and yaw respectively [17].

Consider the general case of the motion of an arbitrary point $P$ in space described by a position vector $\overrightarrow{\boldsymbol{r}}$ of fixed magnitude as shown in Figure 3. The vector undergoes a finite rotation $\overrightarrow{\boldsymbol{\theta}}=\theta \hat{\boldsymbol{n}}$, where $\theta$ is the magnitude of the rotation about an axis having the unit vector $\hat{\boldsymbol{n}}$. After rotation, $P$ has moved to $P^{\prime}$ defined by the position vector $\overrightarrow{\boldsymbol{r}^{\prime}}$ where [18]:

$\overrightarrow{\boldsymbol{r}^{\prime}}=\overrightarrow{\boldsymbol{r}}+(1-\cos \theta)[\hat{\boldsymbol{n}} \times(\hat{\boldsymbol{n}} \times \overrightarrow{\boldsymbol{r}})]+\sin \theta(\hat{\boldsymbol{n}} \times \overrightarrow{\boldsymbol{r}})$

Extending the general case, two consecutive rotations, $\theta_{1}$ and $\theta_{2}$, about axes defined by $\hat{\boldsymbol{n}}_{1}$ and $\hat{\boldsymbol{n}}_{2}$ respectively, are applied to the position vector $\overrightarrow{\boldsymbol{r}}$. The position vector after the second rotation, $\overrightarrow{\boldsymbol{r}^{\prime \prime}}$, can be derived using equation (2). If the sequence of rotations is reversed, however, a different final position vector is obtained because finite rotations of a rigid body about specified axes, although expressed as vectors, do not obey the parallelogram 
law of addition unless the two rotations are about a common axis or are infinitesimal. Assuming infinitesimal rotations the final positions for the two sequences of rotations are equivalent:

$\overrightarrow{\boldsymbol{r}^{\prime \prime}}=\overrightarrow{\boldsymbol{r}}+\left(\theta_{1} \hat{\boldsymbol{n}}_{1}+\theta_{2} \hat{\boldsymbol{n}}_{2}\right) \times \overrightarrow{\boldsymbol{r}}$

The assumption of infinitesimal rotations is valid for typical estimates of shaft angular lateral vibration magnitudes. Additionally, experimentally it is not appropriate to define an order in which to consider the rotations of the shaft about the $X$ - and $Y$-axes. In light of this, the change in position vector, $\Delta \overrightarrow{r_{p}}$, during time increment $\Delta t$, is derived from equation (3). Including a third component, $\overrightarrow{\boldsymbol{\theta}_{\mathbf{z}}}=\theta_{\mathrm{z}} \hat{\mathbf{z}}$, described as the roll or spin about the Z-axis and with a pitch rotation, $\overrightarrow{\boldsymbol{\theta}_{x}}=\theta_{x} \hat{\boldsymbol{x}}$, and a yaw rotation, $\overrightarrow{\boldsymbol{\theta}_{\boldsymbol{y}}}=\theta_{y} \hat{\boldsymbol{y}}$, it is given as:

$\Delta \overrightarrow{\boldsymbol{r}_{\boldsymbol{P}}}=\left(\theta_{x} \hat{\boldsymbol{x}}+\theta_{y} \hat{\boldsymbol{y}}+\theta_{z} \hat{\mathbf{z}}\right) \times \overrightarrow{\boldsymbol{r}_{\boldsymbol{P}}}$

The velocity components of point $P$ due to these rotations can be determined from the time derivative of its position vector:

$\overrightarrow{v_{P}}=\frac{d \overrightarrow{\boldsymbol{r}_{\boldsymbol{P}}}}{d t}=\dot{\theta}_{x}\left(\hat{\boldsymbol{x}} \times \overrightarrow{\boldsymbol{r}_{\boldsymbol{P}}}\right)+\dot{\theta}_{y}\left(\hat{\boldsymbol{y}} \times \overrightarrow{\boldsymbol{r}_{\boldsymbol{P}}}\right)+\dot{\theta}_{z}\left(\hat{\mathbf{z}} \times \overrightarrow{\boldsymbol{r}_{\boldsymbol{P}}}\right)$

However, in practice it is not possible to distinguish between the torsional vibration of the rotating shaft element and the $\theta_{z}$ component of angular lateral vibration. To proceed it is assumed that the motion consists primarily of the rotational velocity, $\omega_{R}(t)$, of the 
illuminated element (which includes the torsional vibration of principal interest). Furthermore, this motion is considered about an axis described by the time-dependent unit vector $\hat{\mathbf{z}}_{\boldsymbol{R}}$ rather than the constant unit vector $\hat{\mathbf{z}}$. The complete velocity of point $P$ can then be considered as the sum of four terms:

$\overrightarrow{\boldsymbol{V}_{\boldsymbol{P}}}=\left(\overrightarrow{\boldsymbol{V}_{x}}+\overrightarrow{\boldsymbol{V}_{y}}+\overrightarrow{\boldsymbol{V}_{z}}\right)+\dot{\theta}_{x}\left(\hat{\boldsymbol{x}} \times \overrightarrow{\boldsymbol{r}_{\boldsymbol{P}}}\right)+\dot{\theta}_{y}\left(\hat{\boldsymbol{y}} \times \overrightarrow{\boldsymbol{r}_{\boldsymbol{P}}}\right)+\omega_{R}\left(\hat{\mathbf{z}}_{\boldsymbol{R}} \times \overrightarrow{\boldsymbol{r}_{\boldsymbol{P}}}\right)$

The first term is the sum of the three components of translation. The second and third terms describe the dependence on angular lateral motion about $X$ - and $Y$-axes, independent of the shaft rotation. In the fourth term, the influence of the main shaft rotation, including the torsional vibration of primary interest, is clear while the time-dependence of $\hat{\mathbf{z}}_{\boldsymbol{R}}$ indicates the second effect of angular lateral vibration, in this case obviously dependent on shaft rotation.

\subsection{DERIVATION OF LTV OUTPUT}

The optical configuration of the LTV to be used in this theoretical development is shown in Figure 4. The original laser beam has been split into two parallel beams by a beam-splitter and mirror and the beams are incident on a shaft of arbitrary cross-section. The shaft is rotating at angular frequency $\omega_{R}(t)$, about the axis defined by $\hat{\mathbf{z}}_{\boldsymbol{R}}(t)$. (In subsequent manipulations, $\omega_{R}$ and $\hat{\mathbf{z}}_{\boldsymbol{R}}$ are used for brevity). The two equal intensity beams are of perpendicular separation $d$ and impinge on the shaft surface at arbitrary points $A$ and $B$, described by position vectors $\overrightarrow{\boldsymbol{r}_{\boldsymbol{A}}}$ and $\overrightarrow{\boldsymbol{r}_{\boldsymbol{B}}}$. These beams are collected in direct backscatter in the direction defined by the unit vector $\hat{\boldsymbol{i}}$. The shaft rotation axis is free to undergo changes of direction but $\hat{\mathbf{z}}_{\boldsymbol{R}}$ is assumed to be identical at each of the incidence points $A$ and $B$. In fact, the analysis assumes $\hat{\mathbf{z}}_{\boldsymbol{R}}$ to be constant from the co-ordinate system origin, $O$, to the furthest 
illuminated shaft cross-section but the instrument's immunity to translational motion makes the choice of the position of the origin an arbitrary one. Without any loss of generality, one possibility is, therefore, to place $O$ in one of the illuminated cross-sections which makes the requirement for constant $\hat{\mathbf{z}}_{\boldsymbol{R}}$ between incidence points $A$ and $B$ a reasonable condition.

The incidence angles defining the relative position of the instrument and target shaft are resolved into two orthogonal planes, as shown in Figure 5. Considering them separately, $\alpha$ is the included angle between $\hat{\boldsymbol{i}}$ and $\hat{\mathbf{z}}$ while $\beta$ is the included angle between the plane formed by the two incident laser beams and the plane of the cross-section of the shaft, perpendicular to the undeflected rotation axis. In the 'ideal' instrument set-up the plane of the LTV beams is parallel to the shaft cross-section, i.e. $\alpha=90^{\circ}$ and $\beta=0^{\circ}$. The $X$-axis of the fixed coordinate system is thus defined as parallel to and in the direction of the laser beam backscatter vector $\hat{\boldsymbol{i}}$ for this special case. For the general set-up detailed in Figure 6, the plane of the laser beams has undergone a rotation about the $X$-axis, changing $\beta$, followed by a rotation about the $Y$-axis, changing $\alpha$.

Each of the laser beams undergoes a Doppler shift when scattered by the moving shaft surface. The beams collected in direct backscatter from points $A$ and $B$ have undergone Doppler shifts $f_{A}$ and $f_{B}$ where [6]:

$$
f_{A, B}=(2 / \lambda) \hat{\boldsymbol{i}} \cdot \overrightarrow{V_{A, B}}
$$

The output from the detector is modulated at the beat frequency: 


$$
f_{\text {beat }}=\left|f_{A}-f_{B}\right|=(2 / \lambda)\left|\hat{\boldsymbol{i}} \cdot\left(\overrightarrow{\boldsymbol{V}_{A}}-\overrightarrow{\boldsymbol{V}_{B}}\right)\right|
$$

Following the format of equation (6), the difference velocity vector term is written:

$$
\hat{\boldsymbol{i}} \cdot\left(\overrightarrow{\boldsymbol{V}_{A}}-\overrightarrow{\boldsymbol{V}_{B}}\right)=\dot{\theta}_{x} \hat{\boldsymbol{i}} \cdot\left[\hat{x} \times\left(\overrightarrow{\boldsymbol{r}_{A}}-\overrightarrow{\boldsymbol{r}_{B}}\right)\right]+\dot{\theta}_{y} \hat{\boldsymbol{i}} \cdot\left[\hat{y} \times\left(\overrightarrow{\boldsymbol{r}_{A}}-\overrightarrow{\boldsymbol{r}_{B}}\right)\right]+\omega_{R} \hat{\boldsymbol{i}} \cdot\left[\hat{\mathrm{z}}_{\boldsymbol{R}} \times\left(\overrightarrow{\boldsymbol{r}_{A}}-\overrightarrow{\boldsymbol{r}_{B}}\right)\right]
$$

Equation (9) demonstrates immunity of the instrument to any translational motion of the shaft which is detected equally by both beams and thus cancels in the difference velocity vector. This insensitivity to radial shaft movement and a similar insensitivity to axial shaft movement was described previously [7-9]. With reference to Figure 6, it is apparent that the position vector terms are equivalent to the vector $\overrightarrow{B A}$ and it is also convenient to re-order the triple scalar products:

$$
\hat{\boldsymbol{i}} \cdot\left(\overrightarrow{\boldsymbol{V}_{A}}-\overrightarrow{\boldsymbol{V}_{B}}\right)=\dot{\theta}_{x} \hat{\boldsymbol{x}} \cdot(\overrightarrow{B A} \times \hat{\boldsymbol{i}})+\dot{\theta}_{y} \hat{\boldsymbol{y}} \cdot(\overrightarrow{B A} \times \hat{\boldsymbol{i}})+\omega_{R} \hat{\mathbf{z}}_{R} \cdot(\overrightarrow{B A} \times \hat{\boldsymbol{i}})
$$

In this expression the first and second terms imply sensitivity of the instrument to angular lateral vibration of the shaft about the orthogonal axes and these components would be detected even if the shaft was not rotating. The third term gives the measurement of shaft rotation speed and, hence, the torsional vibration which is of primary interest. This rotation dependent term is, however, a function of the vector $\hat{\mathbf{z}}_{\boldsymbol{R}}$ and, in subsequent sections, the resulting sensitivity of the instrument to angular lateral vibration will be quantified. Rotation dependent components, superscripted '(1)', and rotation independent components, superscripted '(2)', are considered separately in the following analysis. 
The vector product $(\overrightarrow{B A} \times \hat{\mathbf{i}})$ is obviously important but the shape dependency inherent in $\overrightarrow{B A}$ is inconvenient. On expansion:

$$
(\overrightarrow{B A} \times \hat{\mathbf{i}})=|\overrightarrow{B A}||\hat{\mathbf{i}}| \sin (\delta+\pi / 2) \hat{\boldsymbol{s}}
$$

where $\hat{\boldsymbol{s}}$ is a unit vector perpendicular to both $\overrightarrow{B A}$ and $\hat{\boldsymbol{i}}$ with $(\delta+\pi / 2)$ as their included angle, as shown in Figure 6. It can be seen from the figure that the perpendicular beam separation, $d$, is equal to $|\overrightarrow{B A}| \cos \delta$ and it is also convenient to define a unit vector $\hat{\boldsymbol{d}}=\hat{\boldsymbol{i}} \times \hat{\boldsymbol{s}}$ which is in the direction of a perpendicular line joining the parallel beams as shown in Figure 6. Incorporating these terms allows equation (11) to be re-written as:

$$
(\overrightarrow{B A} \times \hat{\boldsymbol{i}})=d \hat{\boldsymbol{s}}=d \hat{\boldsymbol{d}} \times \hat{\boldsymbol{i}}
$$

Equation (12) clearly demonstrates that the dependence on $\overrightarrow{B A}$, i.e. the shape sensitivity, has been lost allowing the parallel beam arrangement to be used on a shaft of arbitrary crosssection without consequence. Using equation (12), the rotation dependent term from equation (10) can be written in forms that will both be useful for further manipulation as:

$$
\hat{\boldsymbol{i}} \cdot\left(\overrightarrow{\boldsymbol{V}_{\boldsymbol{A}}}-\overrightarrow{\boldsymbol{V}_{B}}\right)^{(1)}=\omega_{R} d\left(\hat{\mathbf{z}}_{\boldsymbol{R}} \cdot \hat{\boldsymbol{s}}\right)=\omega_{R} d \hat{\boldsymbol{d}} \cdot\left(\hat{\mathbf{i}} \times \hat{\mathbf{z}}_{\boldsymbol{R}}\right)
$$

The time-varying shaft rotation vector $\hat{\mathbf{z}}_{\boldsymbol{R}}$ can be expressed as the sum of a constant unit vector $\hat{\mathbf{z}}$ and a fluctuating component $\overrightarrow{\Delta \mathbf{z}}$ related to the angular lateral vibration and this can 
be used to expand the scalar product in equation (13). It is easy to consider the constant component:

$(\hat{\mathbf{z}} . \hat{s})=-\cos \beta \sin \alpha$

but it is useful to express the time-dependent component, $\overrightarrow{\Delta \boldsymbol{z}}$, as the combination of two infinitesimal rotations first:

$\overrightarrow{\Delta \boldsymbol{z}}=\left(\theta_{y}\right) \hat{\boldsymbol{x}}-\left(\theta_{x}\right) \hat{\boldsymbol{y}}$

Combining equations (13) and (15), the variable part equivalent to the scalar product of equation (14) is:

$(\overrightarrow{\Delta \boldsymbol{z}} \cdot \hat{\boldsymbol{s}})=\hat{\boldsymbol{d}} \cdot\left[\left(\hat{\boldsymbol{i}} \times \theta_{y} \hat{\boldsymbol{x}}\right)+\left(\hat{\boldsymbol{i}} \times\left(-\theta_{x}\right) \hat{\boldsymbol{y}}\right)\right]=\left(\theta_{y} \cos \alpha \cos \beta\right)+\left(-\theta_{x} \sin \beta\right)$

Combining equations (13), (14) and (16), the following expression highlights the rotation speed dependent sensitivity of the LTV to angular lateral vibration of the shaft:

$\hat{\boldsymbol{i}} \cdot\left(\overrightarrow{\boldsymbol{V}_{\boldsymbol{A}}}-\overrightarrow{\boldsymbol{V}_{B}}\right)^{(1)}=\omega_{R} d\left[(-\cos \beta \sin \alpha)+\left(\theta_{y} \cos \alpha \cos \beta\right)+\left(-\theta_{x} \sin \beta\right)\right]$

Using equation (12) and evaluating the vector products, the rotation independent part of equation (10) is given by:

$$
\hat{\boldsymbol{i}} \cdot\left(\overrightarrow{\boldsymbol{V}_{\boldsymbol{A}}}-\overrightarrow{\boldsymbol{V}_{\boldsymbol{B}}}\right)^{(2)}=d\left[\dot{\theta}_{x} \cos \beta \cos \alpha+\dot{\theta}_{y} \sin \beta\right]
$$


The resultant beat frequency is finally obtained by substitution of equations (17) and (18) into equation (8). With time-dependent notation again:

$$
\begin{aligned}
f_{\text {beat }}=\frac{2}{\lambda} \mid \omega_{R}(t) d[(-\cos \beta \sin \alpha) & \left.+\left(\theta_{y}(t) \cos \alpha \cos \beta\right)+\left(-\theta_{x}(t) \sin \beta\right)\right] \\
& +d\left[\left(\dot{\theta}_{x}(t) \cos \beta \cos \alpha\right)+\left(\dot{\theta}_{y}(t) \sin \beta\right)\right] \mid
\end{aligned}
$$

This demonstrates the sensitivity of the LTV to all angular motions of the shaft and particularly how the sensitivity to angular lateral vibration depends significantly on the LTV incidence angles, $\alpha$ and $\beta$.

It can be seen from equation (19) that the main factors over which the operator has control in determining the mean beat frequency are the laser beam separation, $d$, and the incidence angles, $\alpha$ and $\beta$. Practical limits to the usable range of $\alpha$ and $\beta$ are determined by the scattering of sufficient light from the target shaft and the minimum value of $f_{\text {beat }}$ which can be demodulated. For one commercial version of the instrument the following angular ranges are quoted when using retro-reflective tape [19]. For side of shaft measurements, a minimum $\alpha$ of $35^{\circ}$ (limited by scattered light intensity) is recommended. For end of shaft measurements a minimum $\alpha$ should be between $30^{\circ}$ (limited by $f_{\text {beat }}$ value) and $55^{\circ}$ (limited by scattered light intensity). Experimentation during this study found similar practical limits and these have been added to figure 9 which will be discussed later. The sensitivity to angular lateral vibration reported in this paper, however, indicates the undesirability of using the instrument at, or even close to, these extremes. Values of $\beta$ up to $75^{\circ}$ are considered acceptable in reducing the effective beam separation which may be necessary, for example, on small diameter shafts, but operation with $\beta \neq 0^{\circ}$ is generally unusual. 
Optimum operation of the instrument is achieved when the plane of the incident laser beams is perpendicular to the rotation axis, then $\alpha=90^{\circ}$ and $\beta=0^{\circ}$ to give:

$$
f_{\text {beat }}=\frac{2}{\lambda}\left|d \omega_{R}(t)\right|
$$

When aligned in this way, sensitivity to $\omega_{R}(t)$ is maximised and angular lateral vibration will have negligible effect on instrument output. Use of the instrument at other incidence angles increases the sensitivity to angular lateral motion and reduces the sensitivity to torsional vibration but such use is often necessary because of restricted access to the measurement location. Additionally, $\alpha$ and $\beta$ can be affected during hand-held use so tripod mounting, while not essential, is preferable.

Conventionally, the LTV is used with $\beta=0^{\circ}$ to maximise the sensitivity of the instrument for the chosen $\alpha$. In this case the measurement derived from a rotating shaft undergoing the motions described is determined from equation (19) as:

$$
f_{\text {beat }}=\frac{2}{\lambda} d\left|\omega_{R}(t)(-\sin \alpha)+\left[\omega_{R}(t) \theta_{y}(t)+\dot{\theta}_{x}(t)\right] \cos \alpha\right|
$$

The second term of this equation indicates the sensitivity of the LTV to angular lateral vibration about both the $X$ - and $Y$-axes, becoming more sensitive to both with decreasing $\alpha$. Obviously this is of concern because the effect on the instrument output will be indistinguishable from torsional vibration and, in what follows, the severity of the sensitivity of the LTV to angular lateral vibration is quantified. 


\subsection{EXPERIMENTAL VALIDATION}

For experimental validation of equation (19) an experimental rig, shown in figure 7, was designed to simulate angular lateral vibration of a rotating shaft. A dynamic shaker was used to drive a section of gear rack in a linear direction. The rack meshed with a large diameter gear, which rotated back and forth to provide the required angular lateral motion about either the $X$ - or $Y$-axis, depending on the relative alignment of the rig and LTV. A small d.c. motor carrying the target shaft was mounted on top of the gear. An a.c. modulation could be added to the d.c. motor drive causing the motor speed to fluctuate, thereby simulating a torsional vibration. The amplitude and frequency of the simulated vibrations and the shaft rotational speed were all independently controllable.

In initial qualitative tests, the LTV was seen to be sensitive to both $\theta_{y}$ and $\theta_{x}$ motions with the magnitude of the former component proportional to mean rotation speed and that of the latter not, as predicted by equation (21). For full quantitative analysis, simulation of $\theta_{y}$ and $\theta_{x}$ motions simultaneously is necessary but this would require a substantially more complicated experimental rig. Simulation of angular lateral motion around one axis is considered sufficient for the validation required here and this has been reported previously $[20,21]$.

To illustrate the sensitivity to angular lateral vibration quantitatively, the LTV was arranged at $\alpha=45^{\circ}$, incident on the side of the shaft. This angle was chosen as a satisfactory compromise of three factors: sensitivity to torsional vibration, sensitivity to angular lateral vibration and to ensure sufficient intensity of light collected. The experimental rig was used to simulate torsional and angular lateral vibration of the shaft. Figure 8 shows the spectrum 
of the LTV signal in which combination of the simulated components at, nominally, $25 \mathrm{~Hz}$ (bending vibration) and $45 \mathrm{~Hz}$ (torsional vibration) is apparent, indicating that it is not possible to distinguish between the motions with this measurement. Guidelines for use of the LTV will now be described before resolution of the combined effects of torsional and bending vibration is offered as further validation of the predicted LTV behaviour.

\subsection{GUIDELINES FOR LTV USE}

A series of engine measurements have been made to estimate typical torsional and bending vibration levels [20, 21]. To aid later comparison, the torsional vibration values are presented in terms of the ratio of torsional vibration, $\Delta \Omega$, to mean speed, $\Omega$. Measurements on a fourcylinder petrol engine, mounted on resilient mounts as in a vehicle, indicated a (second order) maximum $\Delta \Omega / \Omega$ of 0.049 , corresponding to $1420 \mathrm{~m}^{\circ}$, (full load, $1000 \mathrm{RPM}$ ) while the minimum torsional vibration level at any order was estimated to be $\Delta \Omega / \Omega=0.001$. The magnitudes of the harmonic speckle noise peaks [22] present in the LTV output were estimated at $\Delta \Omega / \Omega=3 \times 10^{-4}$, defining the LTV noise-floor. In a further test, two LTVs were set up at different incident angles to measure simultaneously the crankshaft pulley torsional vibration. With one LTV very sensitive to bending vibration, the other only marginally sensitive and assuming equal yaw and pitch amplitudes, an order-of-magnitude estimate of crankshaft pulley bending vibration of $50 \mathrm{~m}^{\circ}$ was made from equation (21). This is consistent with relevant published data [12].

For accurate assessment of torsional vibration, operating arrangements are required in which the sensitivity of the LTV to yaw and pitch vibration is insignificant. The experimental data discussed above are presented in Figure 9 to give a clear indication of their significance. The LTV output and crankshaft pulley angular motion at second order, $\theta_{x}=\theta_{y}=50 \mathrm{~m}^{\circ}$, 
estimated from the simultaneous LTV measurements, can be used to define operating limits for use of the LTV on any shaft experiencing such levels of angular lateral vibration. To allow direct comparison of results, the beat frequency resulting when a steadily rotating shaft undergoes angular lateral vibration is considered to be equivalent to that resulting from a shaft element rotating at a mean speed $\Omega$ with a torsional vibration $\Delta \Omega$. Comparison can therefore be made between the relative magnitudes of angular lateral vibration induced components, genuine torsional vibration and the LTV noise floor, highlighting incidence angles where the sensitivity to angular lateral vibration becomes of concern in this typical application.

It is apparent from Figure 9 that for $\alpha<70^{\circ}$ the magnitude of signal components induced by angular lateral vibration is potentially significant and it is recommended that use of the LTV in this incidence angle range is avoided. In the range $70^{\circ}<\alpha<80^{\circ}$ the error components are of measurable magnitude and caution should be exercised in the interpretation of data. Finally, for $80^{\circ}<\alpha \leq 90^{\circ}$ error components were similar to or smaller than the instrument noise floor and operation of the LTV is effectively immune to all lateral vibration, allowing reliable and accurate measurement of torsional vibration. The same considerations apply to hand-held operation of the LTV because human body movement may induce significant changes in the instrument output in the same way as angular lateral vibration.

\section{MEASUREMENT OF PURE TORSIONAL VIBRATION AND ASSESSMENT OF BENDING VIBRATION}

\subsection{PROCESSING OF LTV OUTPUTS}


Experience in torsional vibration measurement has shown that while it is common to align an LTV with incidence angle $\beta=0^{\circ}$, restricted access often demands use with $\alpha$ for which there is some measurable sensitivity to angular lateral vibrations. It is proposed that with the use of two suitably aligned LTVs, a measure of pure torsional vibration can be derived in situations where a single LTV measurement would leave ambiguity. Simultaneously this technique can give an assessment of the shaft bending vibration [23], a measurement not previously realised by non-contact means. Up to this point the general term angular lateral vibration has been used to describe the angular displacement of a rotating shaft, as this can result from motion of the whole machine or relative motion of the rotating shaft within the machine. The angular lateral vibrations in the engine measurements to follow will, for example, include the whole engine rocking on its mountings as well as crankshaft bending. However, the term bending vibration will now be used to describe the yaw and pitch motions, particularly with respect to engine crankshafts.

In this new configuration two LTVs are arranged symmetrically at equal and opposite incidence angles as shown in Figure 10, on either the side (Arrangement A) or end face (Arrangement $B$ ) of the shaft. With $\beta=0^{\circ}$ in both cases, the sensitivity to bending vibrations is described by equation (21). Figure 11 shows that with a nominally specified incidence angle $\alpha$ there are four possible arrangements. (The LTV outputs for positions 1 and 4 on the side of the shaft are equivalent to that from positions 1' and 4' on the end face of the shaft, the only differences resulting from illumination of a different shaft cross-section). Following the right-hand convention, the four positions are respectively; $+\alpha,(\pi-\alpha),(\pi+\alpha)$ and $-\alpha$. Substituting these angles into equation (21) the following expressions are derived: 


$$
\begin{aligned}
& f_{1}=f_{3}=\frac{2}{\lambda} d\left|\omega_{R}(t) \sin \alpha-\left[\omega_{R}(t) \theta_{y}(t)+\dot{\theta}_{x}(t)\right] \cos \alpha\right| \\
& f_{2}=f_{4}=\frac{2}{\lambda} d\left|\omega_{R}(t) \sin \alpha+\left[\omega_{R}(t) \theta_{y}(t)+\dot{\theta}_{x}(t)\right] \cos \alpha\right|
\end{aligned}
$$

Qualitative experimental investigation confirmed that torsional vibration components were in-phase with each other for all four positions of the LTVs. Referring to Figure 11, the LTV signal components due to $\theta_{y}(t)$ motion (magnitude proportional to rotation speed), relative to position 1, were in-phase at position 3 and out-of-phase at positions 2 and 4, as predicted by equations (22a\&b). Further preliminary tests confirmed the sensitivity of the LTV to $\theta_{x}(t)$ motion (components independent of rotation speed) with the same relative phase relationships as for the $\theta_{y}(t)$ sensitivity.

The proportionality constant for the LTV at position $1, K_{1}$, incorporates the geometrical and optical constants of the arrangement together with the demodulator constant $k_{1 e}$. Therefore the voltage output from this LTV can be written as follows, with similar expressions for the other positions:

$$
V_{1}=K_{1}\left[\omega_{R}(t)-\left(\frac{\omega_{R}(t) \theta_{y}(t)+\dot{\theta}_{x}(t)}{\tan \alpha}\right)\right]
$$

where:

$$
K_{1}=k_{1 e} \frac{2}{\lambda} d \sin \alpha
$$


If simultaneous measurements are taken with two LTVs at positions 1 (or 3) and 2 (or 4), the sum of the outputs provides a measurement of $\omega_{R}(t)$ :

$\frac{V_{1}}{K_{1}}+\frac{V_{2}}{K_{2}}=2 \omega_{R}(t)$

The mean value of this quantity is proportional to the mean rotation speed and the fluctuating component provides the totally unambiguous measure of torsional vibration velocity, immune to all translational and angular lateral shaft vibrations. This arrangement therefore provides a reliable means to achieve accurate measurement of pure torsional vibration from a laterally vibrating shaft where restricted optical access precludes the use of a single LTV with $80^{\circ}<\alpha$ $\leq 90^{\circ}$

The difference in the outputs gives the following signal:

$\frac{V_{1}}{K_{1}}-\frac{V_{2}}{K_{2}}=\frac{2}{\tan \alpha}\left[\omega_{R}(t) \theta_{y}(t)+\dot{\theta}_{x}(t)\right]$

A normalised quantity, derived by combination with equation (24), has also been useful:

$\left(\frac{V_{1}}{K_{1}}-\frac{V_{2}}{K_{2}}\right) /\left(\frac{V_{1}}{K_{1}}+\frac{V_{2}}{K_{2}}\right)=\frac{1}{\tan \alpha}\left[\theta_{y}(t)+\frac{\dot{\theta}_{x}(t)}{\omega_{R}(t)}\right]$

These equations suggest the possibility for simultaneous measurement of the bending vibration of a rotating shaft, a notoriously difficult measurement. However, in this case it is 
not possible to add or subtract signals to give a quantity that is purely dependent on only one

of the orthogonal motions, $\theta_{x}(t)$ or $\theta_{y}(t)$. A measurement obtained in the manner of equation (25a) or (25b) will have sensitivity to both yaw and pitch, with the magnitude of the resultant signal dependent on the relative phase between the components and their individual amplitudes. In some circumstances it may be possible, however, to make a reasonable estimate of the motion and such a case is made at the end of section 3.3

\subsection{EXPERIMENTAL VALIDATION}

The experimental rig described previously and shown in figure 7 was used to simulate the vibrations of a shaft. An accelerometer was fixed to the rack which rotated the gear wheel, providing the "true" measure of the simulated shaft bending vibration. The "true" simulated speed fluctuation measurement was provided by a third LTV set with $\alpha=90^{\circ}$ such that this measurement was effectively insensitive to the bending vibration of the shaft.

Two LTVs were arranged to provide the sum and difference functions described by equations (24) and (25). For this experimental study only yaw, $\theta_{y}(t)$, was simulated for proof of the principle described. In this case, measurement of the bending motion can be achieved if the two LTVs are aligned with their beams incident in either the $X-Z$ or $Y-Z$ plane. For the validation the $X-Z$ plane was used.

Figure 8 showed the spectrum of an LTV measurement demonstrating indistinguishable sensitivity to both torsional and bending vibration. A second LTV, aligned at an equal and opposite angle and incident on same shaft element, can be used with the first LTV to obtain the measurement of pure torsional vibration. Figure 12a shows the torsional vibration resolved in accordance with equation (24) which demonstrates excellent agreement with the 
"true" measurement in Figure 12b. This technique therefore provides a measure of pure torsional vibration in situations where measurement with a single LTV would be ambiguous due to sensitivity to shaft bending vibration.

With the bending motion constrained to $\theta_{y}(t)$, equation (25b) provides an exact measure of this angular vibration:

$\left(\frac{V_{1}}{K_{1}}-\frac{V_{2}}{K_{2}}\right) /\left(\frac{V_{1}}{K_{1}}+\frac{V_{2}}{K_{2}}\right)=\frac{\theta_{y}(t)}{\tan \alpha}$

Figure 13a shows the bending vibration measurement resolved in accordance with equation (26) and Figure 13b shows the close agreement between the resolved and "true" measurements, including the presence of relatively small harmonics near $50 \mathrm{~Hz}$ and $75 \mathrm{~Hz}$.

The sensitivity of the technique to bending vibrations can be improved if required. This is achieved by decreasing the incidence angle $\alpha$ and usually necessitates that the LTVs are incident on the end face of the shaft as in Arrangement B of Figure 10. Experimental measurements from the end face of the shaft with $\alpha=15^{\circ}$ demonstrated the minimum measurable bending vibration to be of the order of $1 \mathrm{~m}^{\circ}$. Additionally, the signal to noise ratio for bending vibration measurements is improved by reducing the incidence angle. As for torsional vibration measurements using this technology, when studying bending vibrations at integer multiples of rotation speed, the lower limit of measurement is defined by the level of speckle noise harmonics [22].

\subsection{DIESEL ENGINE CRANKSHAFT VIBRATION}


A loaded 2.0 litre diesel engine was used as a first practical example to demonstrate the measurement of pure torsional vibration with estimation of crankshaft bending vibration. Two LTVs were arranged symmetrically at approximately $\alpha=35^{\circ}$ on the end face of the crankshaft pulley. With this alignment measurement by a single instrument would be contaminated by bending vibration. A third LTV was set up at $\alpha=75^{\circ}$ (limited by access), an arrangement virtually insensitive to shaft bending vibration, to provide the "true" measure of torsional vibration for comparison. The first two LTV outputs were recorded simultaneously and processed to give the resolved torsional and bending vibration measurements. (Some differences between the measurements may be attributed to the fact that it was not possible to obtain the results from all three LTVs simultaneously).

Figure 14 shows a typical torsional vibration signal measured on the engine at 750rpm, from a single LTV at $\alpha=35^{\circ}$. At this rotation speed the 2nd order crankshaft torsional vibration dominates the response. Figures 15a-c show the torsional vibration spectra from a single LTV at $\alpha=35^{\circ}$, from the "true” measurement and from the resolved signal respectively. The main orders of torsional vibration, namely, 2nd, 4th and 6th, are clearly evident in each case and comparison of these spectra highlights important differences.

Firstly, it is apparent that the magnitudes of the resolved measurement components are closer to the "true" measurement than those of the single LTV. In addition, the response in the frequency range $300 \mathrm{~Hz}$ to $500 \mathrm{~Hz}$ is quite different between the resolved signal and that from the single LTV. This section of the spectrum has been expanded in Figures 16a\&b for the single LTV and resolved measurement respectively. The spectra are significantly different and it will be shown shortly how this is due to the substantial contribution of bending vibration in the single LTV measurement. The resolved measurement has a clear peak at 
$375 \mathrm{~Hz}$ at which there is known to be a torsional mode of the crankshaft. This mode cannot be distinguished in Figure 16a in which a large peak at $400 \mathrm{~Hz}$ appears to dominate. Comparison of the figures highlights the technique's importance for accurate assessment of torsional vibration.

Using equation (25b), the crankshaft bending vibration is shown in Figure 17, differing significantly, but in the manner expected, from the torsional vibration signal of Figure 14. The repeating pattern of impacts from the firing of the four cylinders is clear and occurs over a two revolution time period as expected for a four-stroke engine. The largest impact is that of cylinder 1 , closest to the pulley position, consistent with previous experimental studies of crankshaft behaviour $[13,14,24]$. Minor variations between impact sequences occur as a result of combustion cyclic variability. However, as discussed previously, this measurement is sensitive to both yaw and pitch motions and resolution of individual motions is not yet possible.

The spectrum of this motion is shown in Figure 18 with significant differences from the shape of the torsional vibration spectrum in Figure 15c, as expected. In addition to the distinct bending vibration peaks occurring at the first four orders of rotation, the spectrum shows a large vibration response in the $350-500 \mathrm{~Hz}$ range, which was seen previously to corrupt the measurement from the single LTV at $\alpha=35^{\circ}$. It is proposed that the broad peak centred at $455 \mathrm{~Hz}$ is the first bending mode of the crankshaft, excited by the impacts of the cylinders firing. Although analytical modelling of crankshaft response has been comprehensive, previous experimental measurements of crankshaft bending on a rotating engine have been limited due to a lack of suitable measurement techniques. However, comparable values of crankshaft bending mode frequencies have been reported for similarly 
sized engines [13, 24]. In basic modal analysis of the stationary engine crankshaft, the natural frequency of the crankshaft in bending was found to be in the range $456-476 \mathrm{~Hz}$, depending on the angular position of the crankshaft and con-rods. Results recorded for five speeds of the engine under load showed a similar response in the $350-500 \mathrm{~Hz}$ range with similar signal magnitude. The dominant peak at $400 \mathrm{~Hz}$ in figure $16 \mathrm{a}$, described earlier, seems, therefore, to be due to a combination of torsional and bending motions and may not, itself, be a particularly significant vibration component.

Figure 19 shows the measurement, band-pass filtered around this crankshaft bending resonant frequency. From equations (25a\&b), the resultant measurement is seen to depend on both the $\theta_{y}(t)$ and $\theta_{x}(t)$ motions. Assuming equal magnitudes of each motion, at higher frequencies the 'velocity' component, $\left|\dot{\theta}_{x}(t)\right|=\omega_{x}\left|\theta_{x}(t)\right|$, will dominate the response. If all the signal is assumed to result from $\dot{\theta}_{x}(t)$ sensitivity, the peak pitch of the crankshaft pulley in this mode is estimated to be $12 \mathrm{~m}^{\circ}$ with modal damping approximately $5 \%$ of critical. Modal damping, in particular, is a difficult but important parameter to estimate and the proposed measurement system represents significant progress in enabling this estimate.

\section{CONCLUSIONS}

The measurement of torsional and bending vibration from rotating shaft systems has been addressed by significant new developments in experimental techniques. Comprehensive theory has been derived for the operation of the laser torsional vibrometer (LTV) to account for the effect of all shaft motions on the measurement obtained. Whilst inherently immune to translational target vibration, the LTV is sensitive to angular lateral vibration of the target 
shaft which contributes to the measurement derived and is indistinguishable from the intended measurement of torsional vibration. The effect is dependent on the incidence angle of the LTV relative to the shaft, becoming more severe as the instrument is moved away from the optimum arrangement, as is often required by access restriction. The predicted sensitivity of the instrument to angular lateral motion was confirmed experimentally, clearly demonstrating the potential for ambiguity in a measurement from a single LTV. Guidelines were specified to ensure effective immunity to all lateral motion in a typical engine application.

It was recommended that use of the instrument with the incidence angle $\alpha<70^{\circ}$ is avoided, while in the range $80^{\circ}<\alpha \leq 90^{\circ}$ error components were similar to or smaller than the instrument noise floor and operation is effectively immune to angular lateral motion. Emphasis was given to the desirability of using the LTV with $\alpha \approx 90^{\circ}$.

For situations where optimum alignment is not possible, a new and reliable technique was introduced which provides an accurate measure of pure torsional vibration in situations where a single LTV demonstrates unacceptable sensitivity to angular lateral vibrations. Two symmetrically aligned LTVs are used, with the measurement of pure torsional vibration derived from addition of their output signals. Simultaneously this technique can provide noncontact assessment of angular lateral, or bending, vibration directly from a rotating component.

In a diesel engine application, comparison of the genuine torsional vibration with that measured from a single LTV identified discrepancies due to the effects of shaft bending vibration. Furthermore, the bending vibration signal gave a clear indication of the shaft 
motion due to the combustion impacts. From the spectrum of this measurement it was possible to locate the natural frequency of the crankshaft in bending, excited by the firing impulses, and this was confirmed in further modal analysis tests on the engine. During operation, the magnitude of the bending motion of the crankshaft pulley and the damping for this mode could be estimated.

However, obtaining the bending vibration measurement from the difference of two LTV measurements, in the manner described, is sensitive to angular lateral shaft motion about both the $X$ - and $Y$-axis. A possible, partial solution to this problem is to take two such measurements simultaneously, with alignment of one LTV pair around the $X$-axis as in this paper and the second pair equivalently around the $Y$-axis, resolving the pitch and yaw by mathematical means. This could be done in a similar manner to that described for measurement of the radial vibration of a rotating shaft with the use of translational laser vibrometers [25] where similar cross-sensitivity problems exist.

These developments in laser vibrometry for non-contact measurements of shaft vibration will allow a much greater depth of study to be realised in the design and development of rotating machinery. The assessment of shaft bending vibration, in particular, is a further step forward in the application of laser vibrometry to rotating machinery diagnostics.

\section{REFERENCES}

1 R.L. SMITH 1992 Sound and Vibration 26 (September) 22-24. Torsional vibration. 
2 R.N. BROWN 1960 Transactions of the ASME: Journal of Engineering for Power 82 215-220. A torsional vibration problem as associated with synchronous motor driven machines.

3 J.M. VANCE 1988 Rotordynamics of Turbomachinery. New York: John Wiley \& Sons.

4 K. FUJII 1991 Proceedings of the $6^{\text {th }}$ International Pacific Conference on Automotive Engineering Seoul, South Korea. 357-363. SAE Paper 912495. A simulation of crankshaft bending vibration.

5 T. KAMIYA, T. ATSUMI and K. TASAKA 1988 SAE Transactions 97(4) 4.59-4.65. SAE Paper 880078. Toyota's new type of crankshaft pulley to improve the compartment tone quality.

6 L.E. DRAIN 1980 The Laser Doppler Technique Ch.9. Chichester: John Wiley \& Sons.

7 N.A. HALLIWELL. C.J.D. PICKERING and P.G. EASTWOOD 1984 Journal of Sound and Vibration 93(4) 588-592. The laser torsional vibrometer: a new instrument.

8 N.A. HALLIWELL and P.G. EASTWOOD 1985 Journal of Sound and Vibration 101(3) 466-499. The laser torsional vibrometer.

9 N.A. HALLIWELL 1996 Journal of Sound and Vibration 190(3) 399-418. The laser torsional vibrometer: a step forward in rotating machinery diagnostics.

10 J.M. VANCE and R.S. FRENCH 1986 Transaction of the ASME Journal of Mechanisms, Transmission and Automation in Design 180 565-577 Measurement of Torsional Vibration in Rotating Machinery.

11. BRÜEL \& KJÆR (N.A. Halliwell, S.J. Rothberg, T.J. Miles, P.G. Eastwood, C.J.D. Pickering and K. Gatzwiller) 1994 On the Working Principle of Torsional Vibration Meter Type 2523: Application Note. Denmark. 
12 M.F. ALBRIGHT and D.F. STAFFELD Proceedings of the 1991 Noise and Vibration Conference Transverse City, USA. Society of Automotive Engineers P-244, 291-305. SAE Paper 911073. Noise and vibration refinement of the Ford 3.8 litre powertrain.

13 M.KINOSHITA, T. SAKAMOTO and H.OKAMURA 1989 SAE Transactions 98(3) 1605-1615. SAE Paper 891127. An experimental study of a torsional/bending damper pulley for an engine crankshaft.

14 O. KURODA and Y. FUJII 1988 SAE Transactions 97(4) 4.73-4.82. SAE Paper 880083. An approach to improve engine sound quality.

15 Y-S. PARK, J-Y PARK and C-W LEE 1986 SAE Transaction 95(1) 1.1157-1165. SAE Paper 860232. Dynamic torsional and bending stress measurement from operating crankshaft.

16 N.A. HALLIWELL, A. HOCKNELL and S.J. ROTHBERG, 1997, Journal of Sound and Vibration, 208(3), 1997, 497-500. On the Measurement of Angular Vibration Displacements: A Laser Tiltmeter.

17 R.A. COLLACOTT 1979 Vibration Monitoring and Diagnosis Ch. 2. London: George Goodwin.

18 J.H. WILLIAMS 1996 Fundamentals of Applied Dynamics. New York: John Wiley \& Sons.

19 BRÜEL \& KJÆR 1992 Torsional Vibration Meter Type 2523 - Instruction Manual. Denmark.

20 T.J. MILES, M. LUCAS and S.J. ROTHBERG 1995 Proceedings of the 15th ASME Biennial Conference on Vibration and Noise Boston USA. 84-3(C) 1451-1460. The laser torsional vibrometer: successful operation during lateral vibrations.

21 T.J. MILES, M. LUCAS and S.J. ROTHBERG 1996 Automotive Refinement (IMechE Seminar Proceedings: Autotech 95. Birmingham, UK.) 65-73 Paper C498/26/041. The laser 
torsional vibrometer: optimum use in the presence of lateral vibrations. London: Mechanical Engineering Publications.

22 S.J. ROTHBERG, J.R. BAKER and N.A. HALLIWELL 1989 Journal of Sound and Vibration 135(3) 516-522. Laser vibrometry: pseudo-vibrations.

23 T.J. MILES, M. LUCAS and S.J. ROTHBERG 1996 Optics Letters 21(4) 296-298. Bending vibration measurement on rotors by laser vibrometry.

24 S-K. LEE, B-U. CHOI and S-D. YEO 1993 New Engine Design and Engine Component Technology Society of Automotive Engineers SP-972, 11-21. SAE Paper 930618. Identification of the relation between crankshaft bending and interior noise of $\mathrm{A} / \mathrm{T}$ vehicle in idle state.

25 S.J. ROTHBERG and N.A. HALLIWELL 1994 Transaction of the ASME: Journal of Vibration and Acoustics 116 326-331. Vibration measurements on rotating machinery using laser Doppler velocimetry. 


\section{TORSIONAL AND BENDING VIBRATION MEASUREMENT ON ROTORS}

USING LASER TECHNOLOGY

\section{T.J. MILES, M. LUCAS, N.A. HALLIWELL and S.J. ROTHBERG}

\section{FIGURES}

Figure 1. Rigid-rotor lateral vibration modes for a symmetrical rotor.
a. Cylindrical whirl orbit.
b. Conical whirl orbit.

Figure 2. General motion of a point on a rotating shaft.

Figure 3. Displacement of point $\mathrm{P}$ due to finite rotation.

Figure 4. Optical geometry of the laser torsional vibrometer.

Figure 5. Definition of the LTV target shaft incidence angles.
a. Plan view.
b. Front view.

Figure 6. Arrangement of vectors describing instrument laser beams and target geometry.

Figure 7. Experimental rig for simulation of angular lateral vibration.

Figure 8. LTV output spectrum showing sensitivity to torsional and bending vibration.

Figure 9. Guidelines for successful operation of the LTV with minimisation of lateral vibration errors.

Figure 10. Arrangements of two LTVs for measurement of torsional vibration immune to angular lateral vibration.

Figure 11. Angular positions of the LTVs around the shaft in the $X-Z$ plane

Figure 12. Torsional vibration measurements immune to bending vibration.

a. Resolved data from two LTVs. 
b. Independently measured data.

Figure 13. Bending vibration measurements
a. Resolved data from two LTVs.
b. Independently measured data.

Figure 14. Torsional vibration time trace measurement from the crankshaft of a diesel engine running under load at 750rpm with a single LTV at $\alpha=35^{\circ}$.

Figure 15. Torsional vibration spectra from diesel engine crankshaft at 750rpm.
a. Single LTV at $\alpha=35^{\circ}$.
b. Independent LTV at $\alpha=75^{\circ}$ (“true” measurement).
c. Resolved measurement of pure torsional vibration.

Figure 16. Expanded view of spectra in Figure 14 (Diesel engine crankshaft at 750rpm).
a. $\quad$ Single LTV at $\alpha=35^{\circ}$.
b. Resolved measurement of pure torsional vibration.

Figure 17. Bending vibration measurement of diesel engine crankshaft under load at 600rpm.

Figure 18. Spectrum of diesel engine crankshaft bending vibration.

Figure 19. Filtered time history of crankshaft bending vibration. 


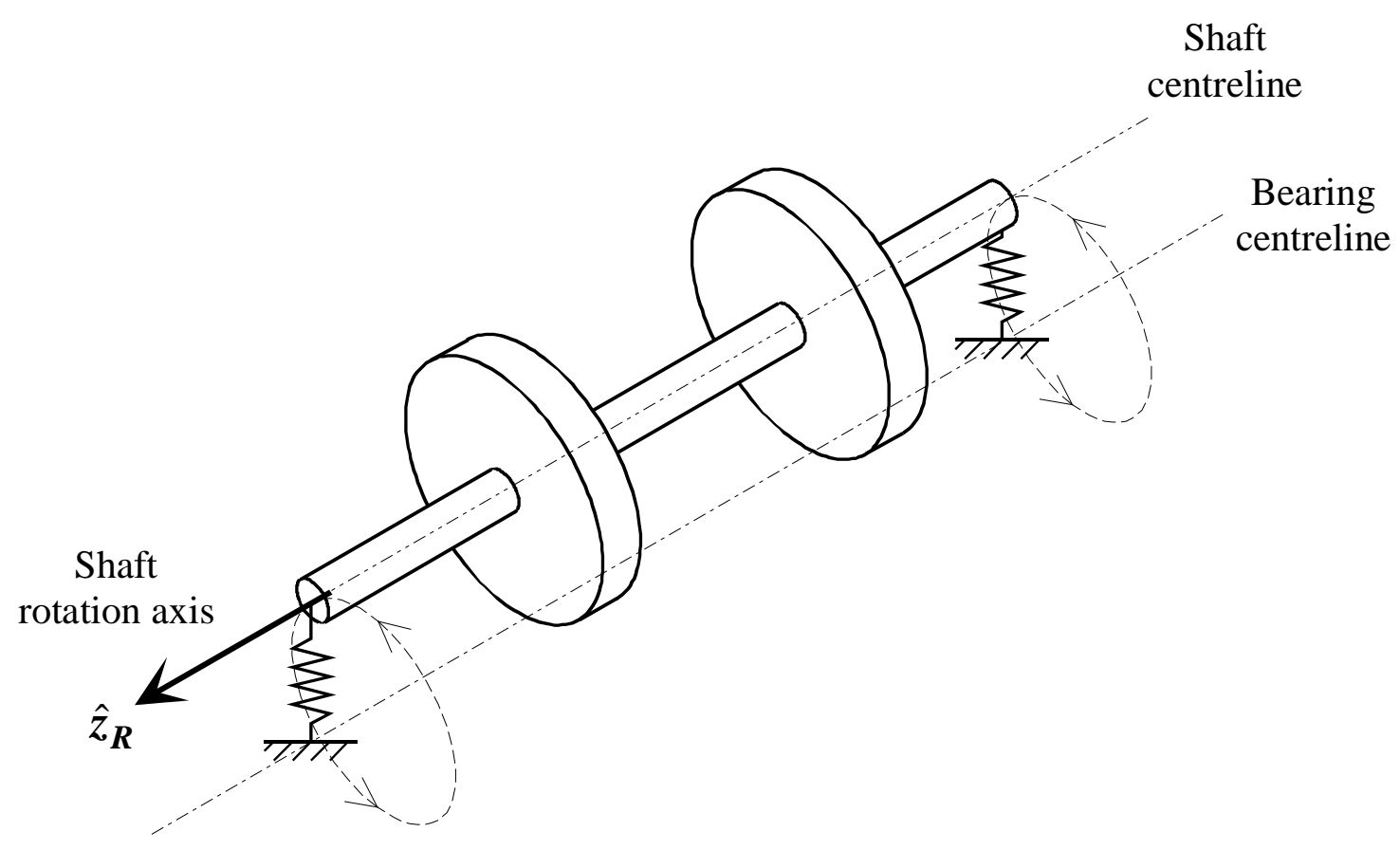

(a)

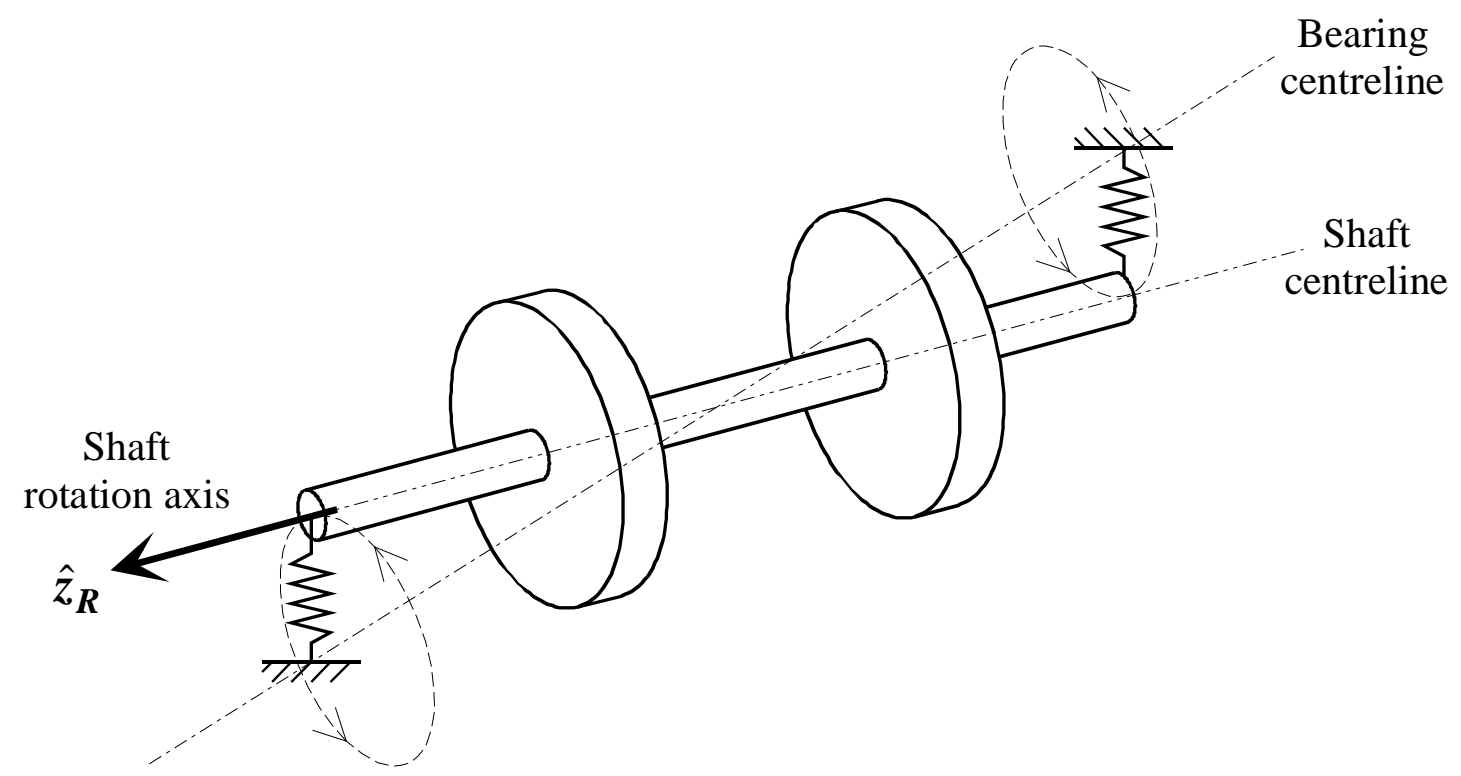

(b)

Figure 1

Torsional and Bending Vibration Measurement on Rotors using Laser Technology

T.J. Miles, M. Lucas, N.A. Halliwell and S.J. Rothberg Loughborough University 


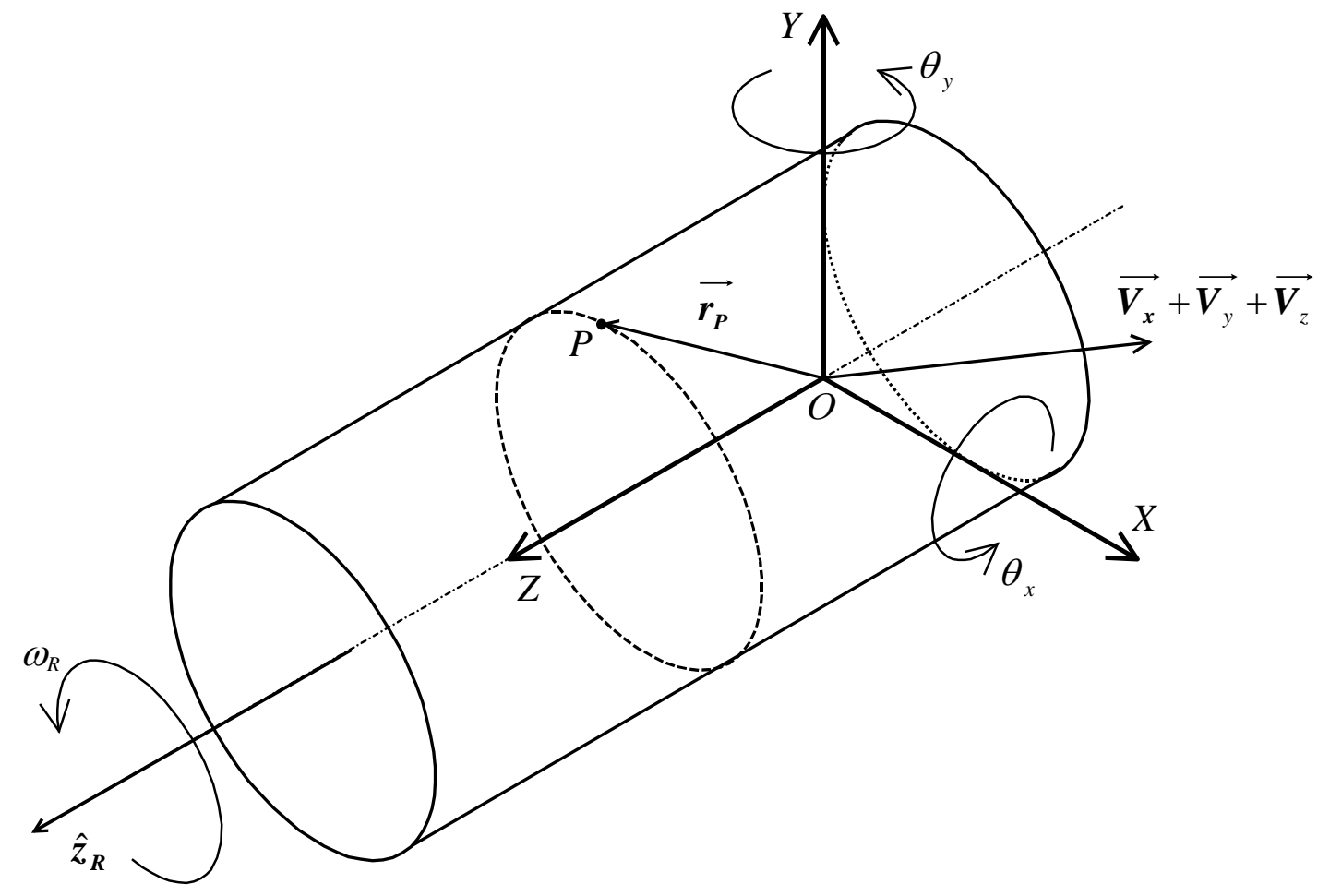

Figure 2

Torsional and Bending Vibration Measurement on Rotors using Laser Technology

T.J. Miles, M. Lucas, N.A. Halliwell and S.J. Rothberg Loughborough University 


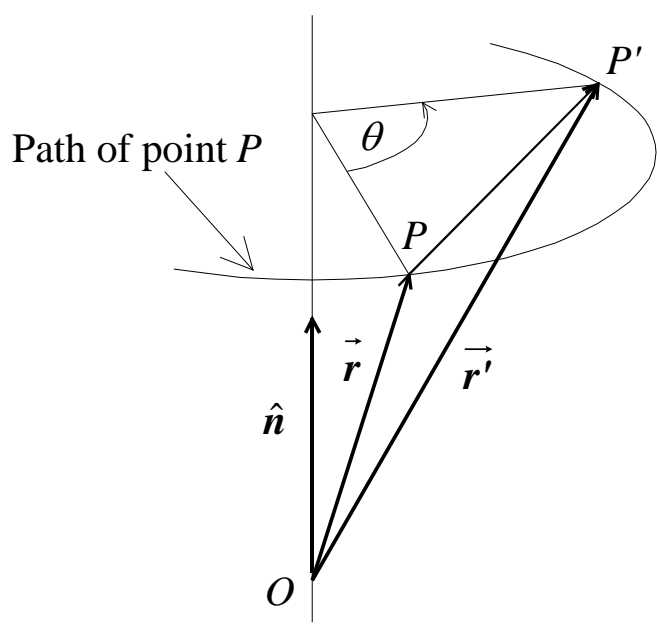

Figure 3

Torsional and Bending Vibration Measurement on Rotors using Laser Technology

T.J. Miles, M. Lucas, N.A. Halliwell and S.J. Rothberg

Loughborough University 


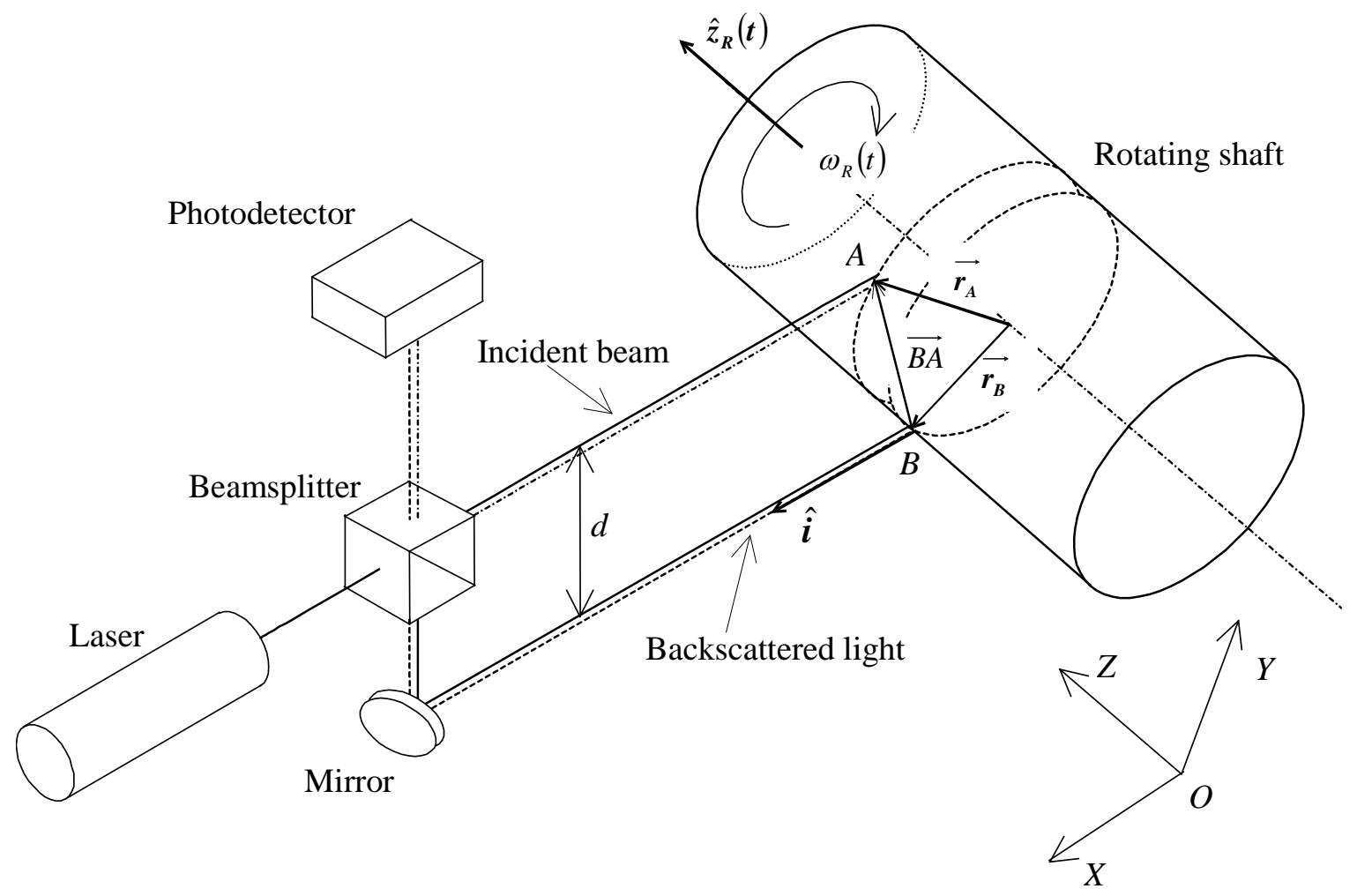

Figure 4

Torsional and Bending Vibration Measurement on Rotors using Laser Technology

T.J. Miles, M. Lucas, N.A. Halliwell and S.J. Rothberg

Loughborough University 


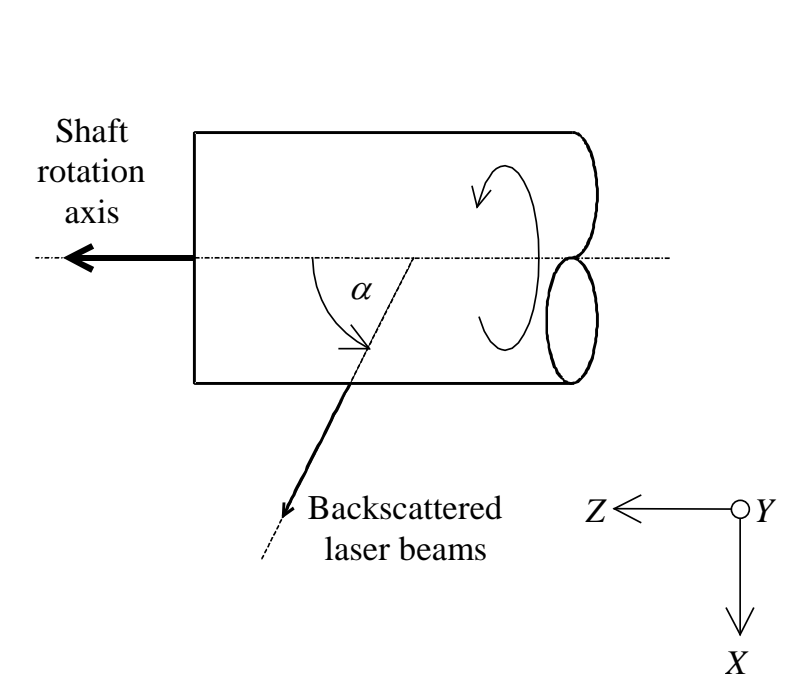

a. Plan view

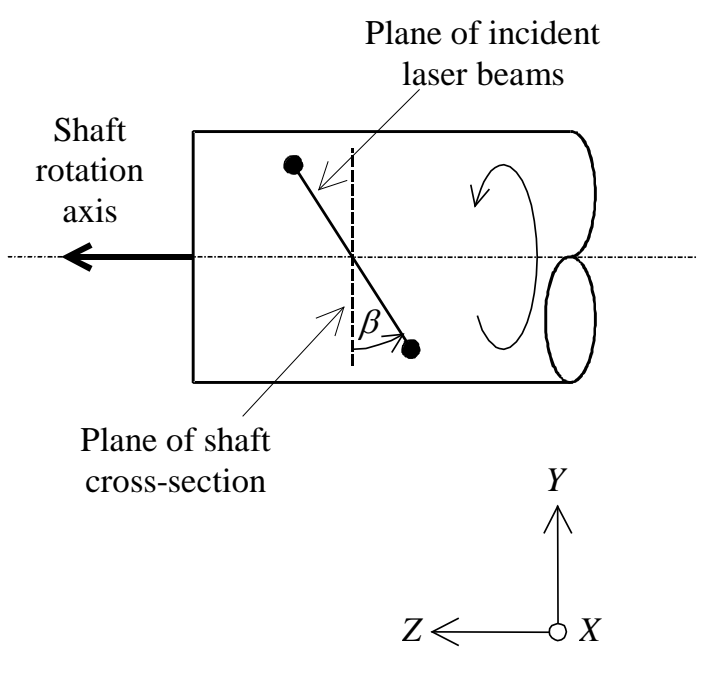

b. Front view

Figure 5

Torsional and Bending Vibration Measurement on Rotors using Laser Technology

T.J. Miles, M. Lucas, N.A. Halliwell and S.J. Rothberg Loughborough University 


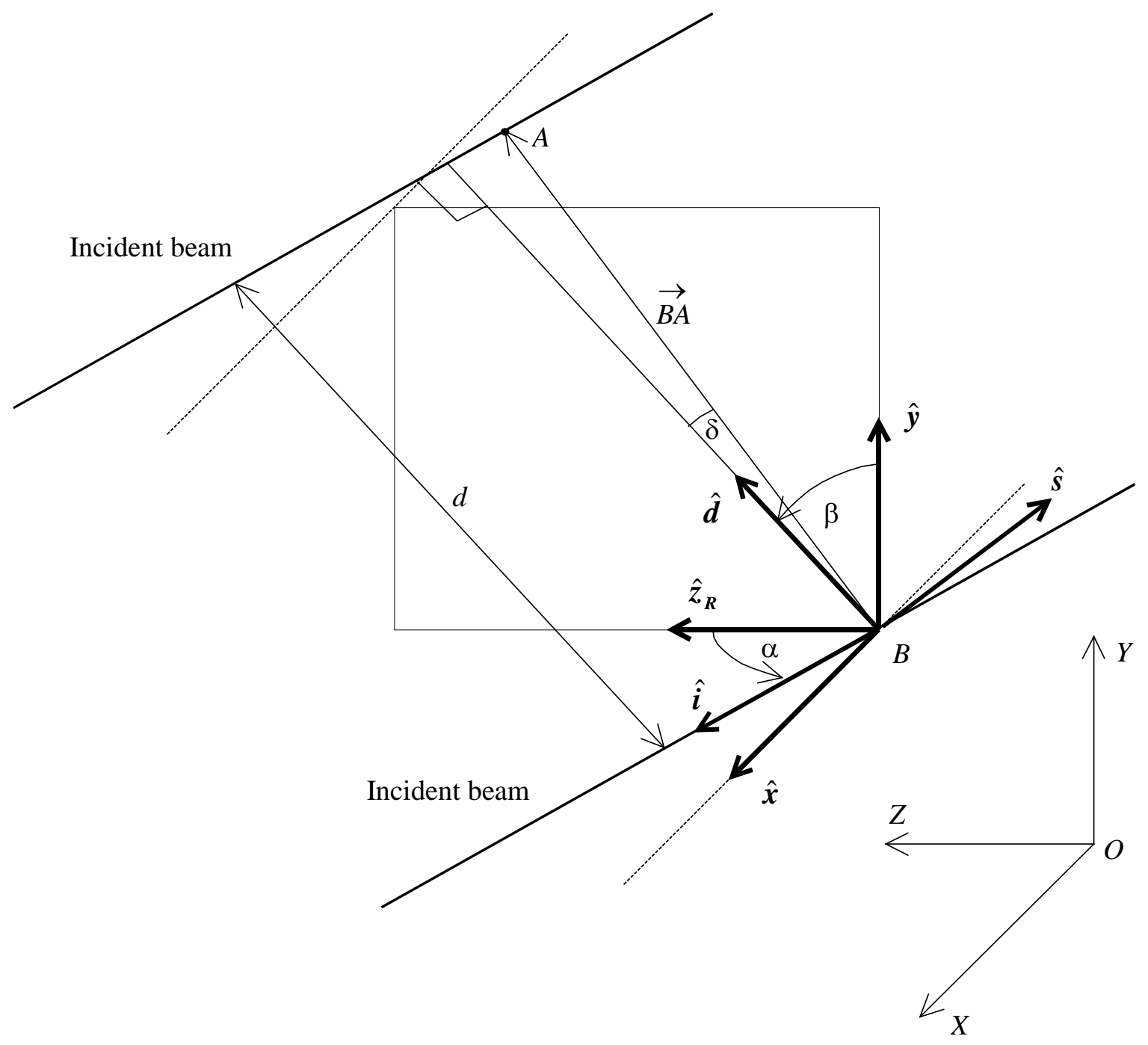

Figure 6

Torsional and Bending Vibration Measurement on Rotors using Laser Technology

T.J. Miles, M. Lucas, N.A. Halliwell and S.J. Rothberg

Loughborough University 
Figure 7

Torsional and Bending Vibration Measurement on Rotors using Laser Technology

T.J. Miles, M. Lucas, N.A. Halliwell and S.J. Rothberg Loughborough University 


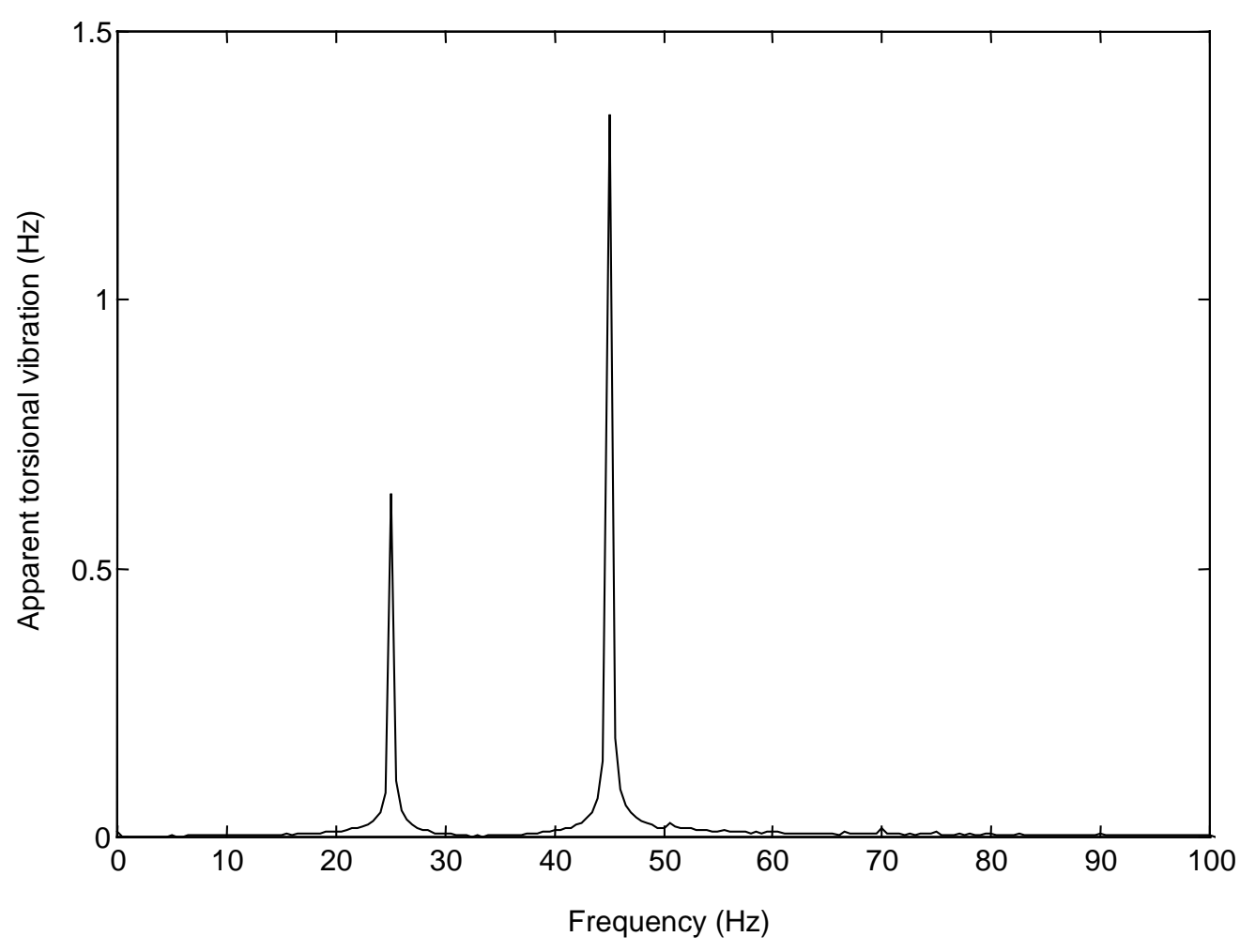

Figure 8

Torsional and Bending Vibration Measurement on Rotors using Laser Technology

T.J. Miles, M. Lucas, N.A. Halliwell and S.J. Rothberg Loughborough University 


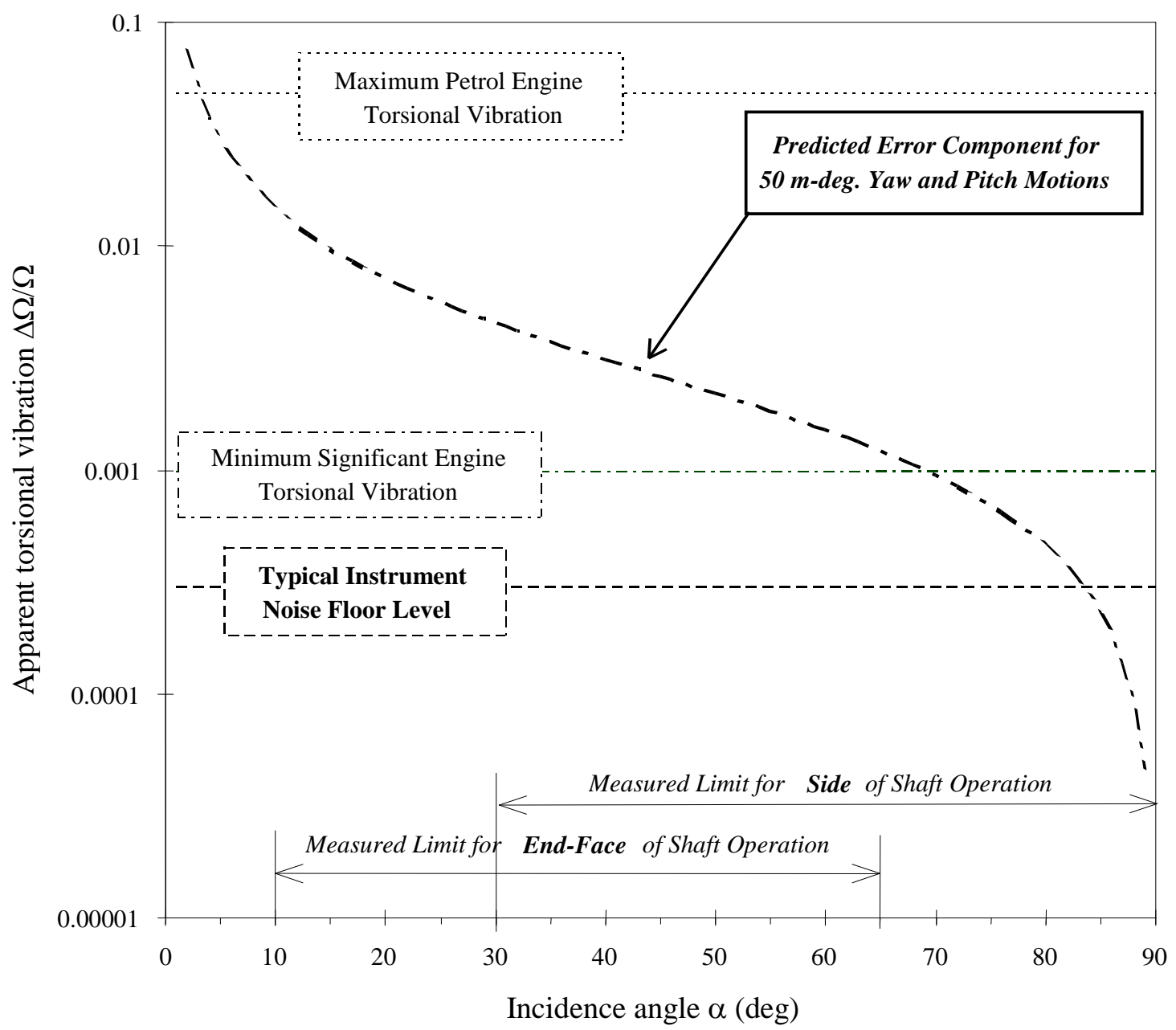

Figure 9

Torsional and Bending Vibration Measurement on Rotors using Laser Technology

T.J. Miles, M. Lucas, N.A. Halliwell and S.J. Rothberg Loughborough University 


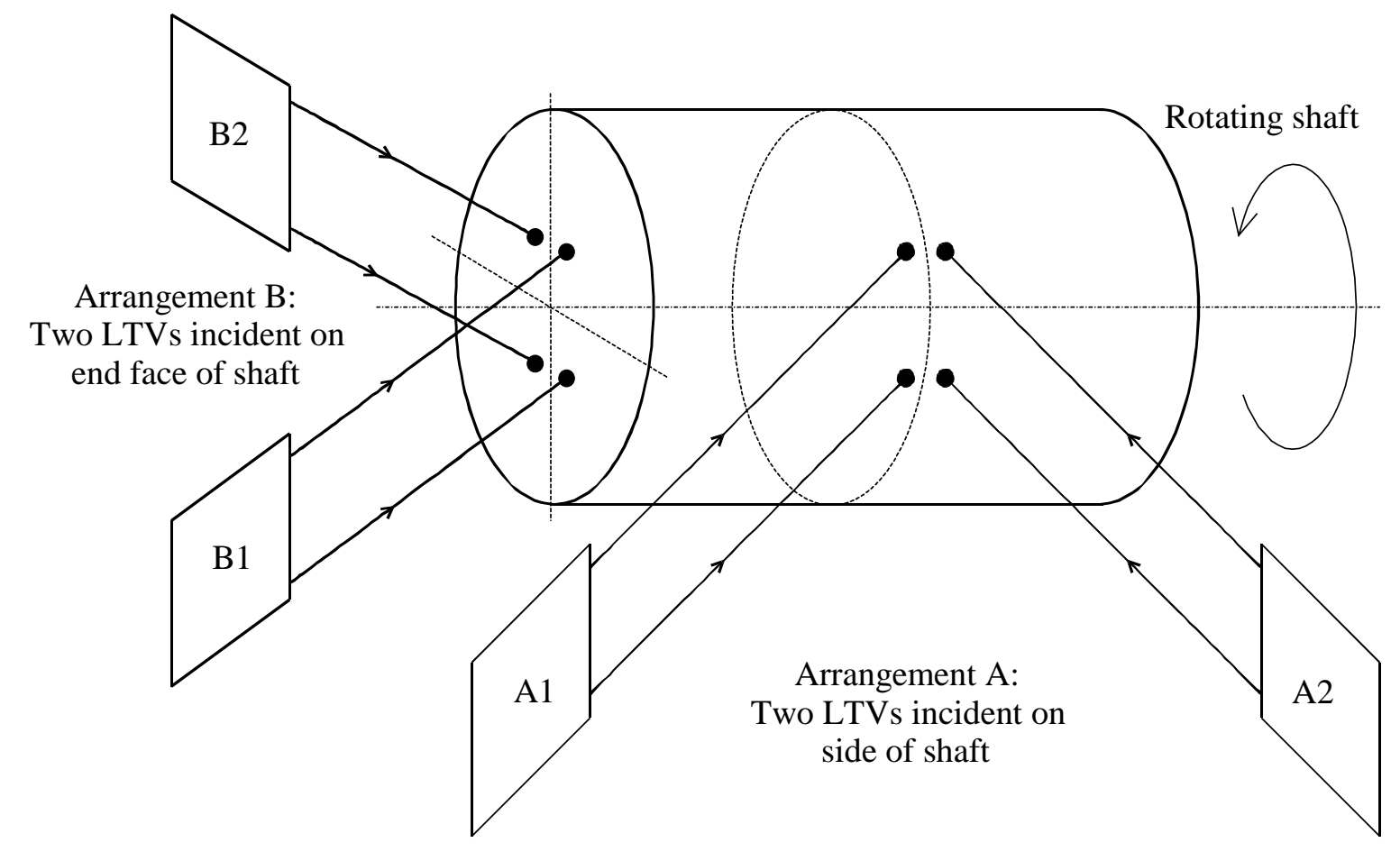

Figure 10

Torsional and Bending Vibration Measurement on Rotors using Laser Technology

T.J. Miles, M. Lucas, N.A. Halliwell and S.J. Rothberg

Loughborough University 


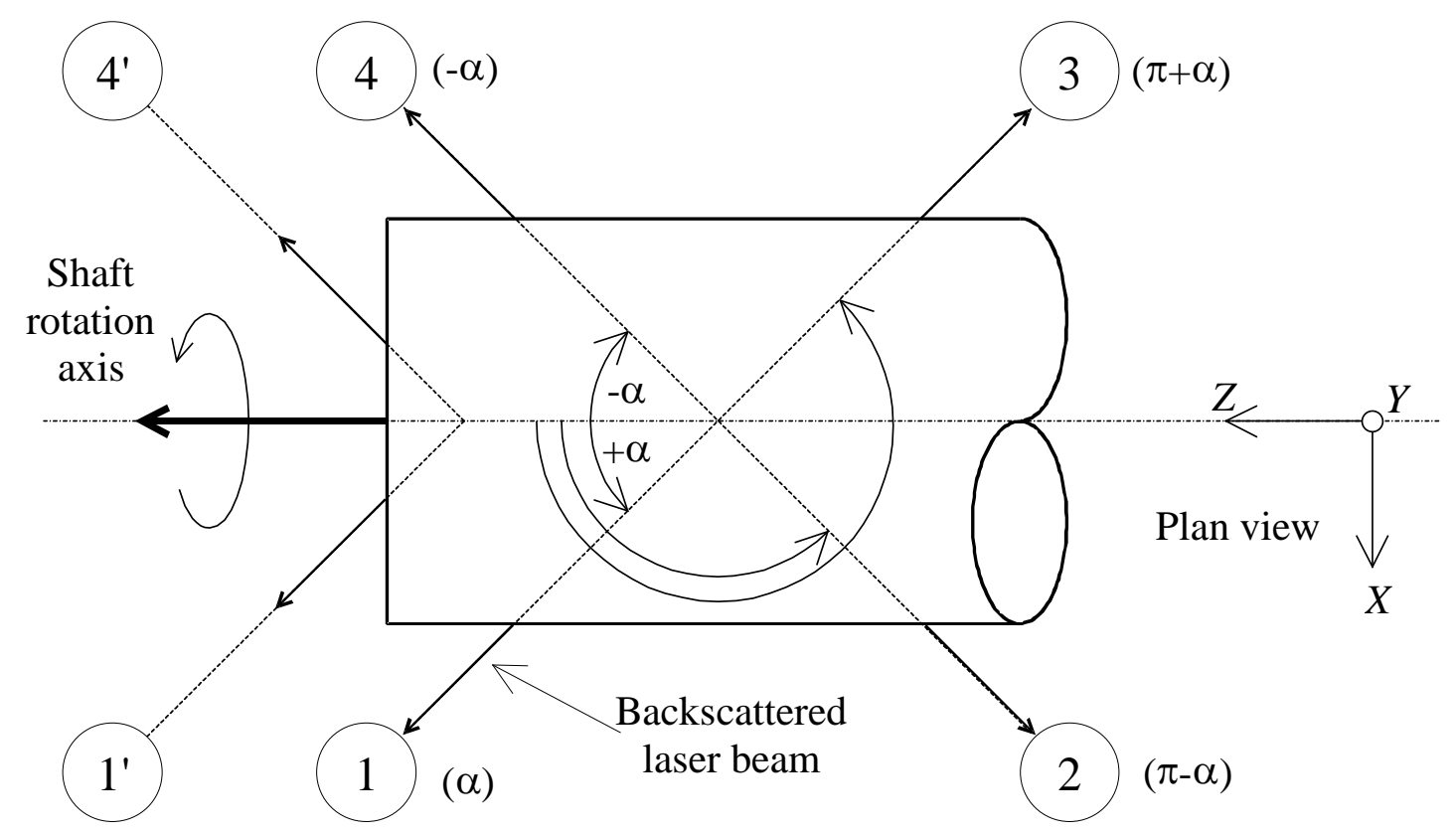

Figure 11

Torsional and Bending Vibration Measurement on Rotors using Laser Technology

T.J. Miles, M. Lucas, N.A. Halliwell and S.J. Rothberg Loughborough University 

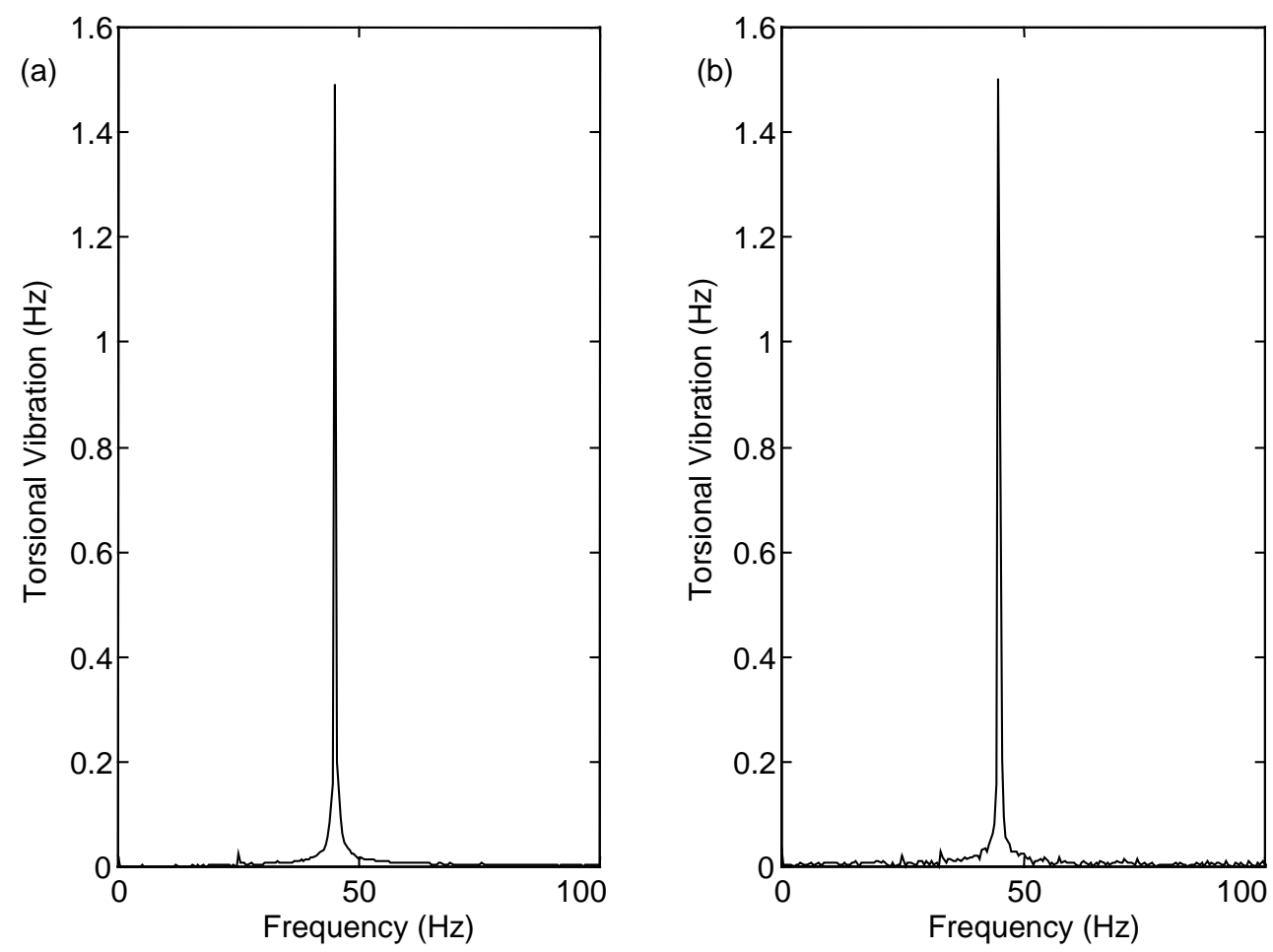

Figures 12a\&b

Torsional and Bending Vibration Measurement on Rotors using Laser Technology

T.J. Miles, M. Lucas, N.A. Halliwell and S.J. Rothberg Loughborough University 

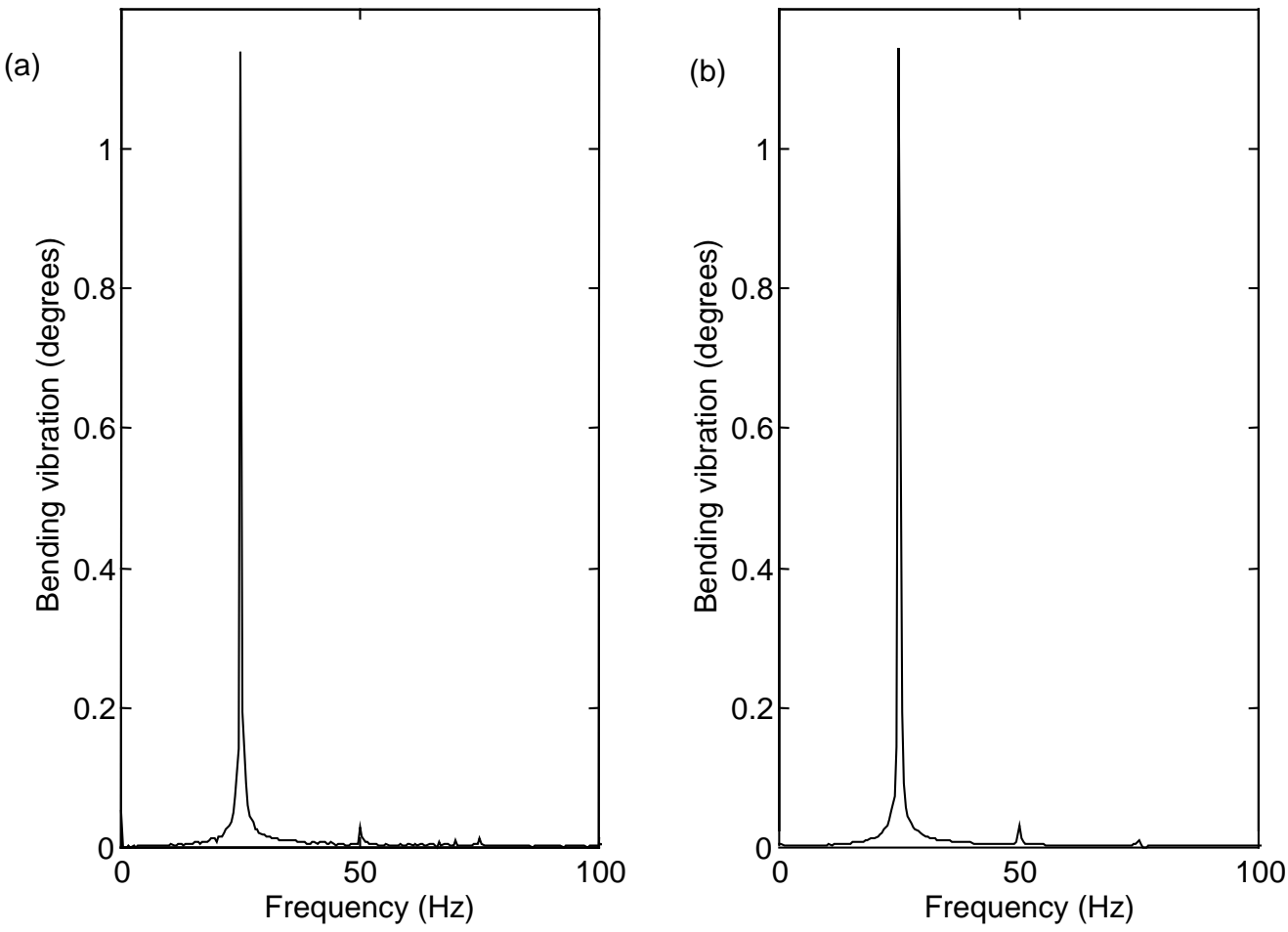

Figure 13a\&b

Torsional and Bending Vibration Measurement on Rotors using Laser Technology

T.J. Miles, M. Lucas, N.A. Halliwell and S.J. Rothberg Loughborough University 


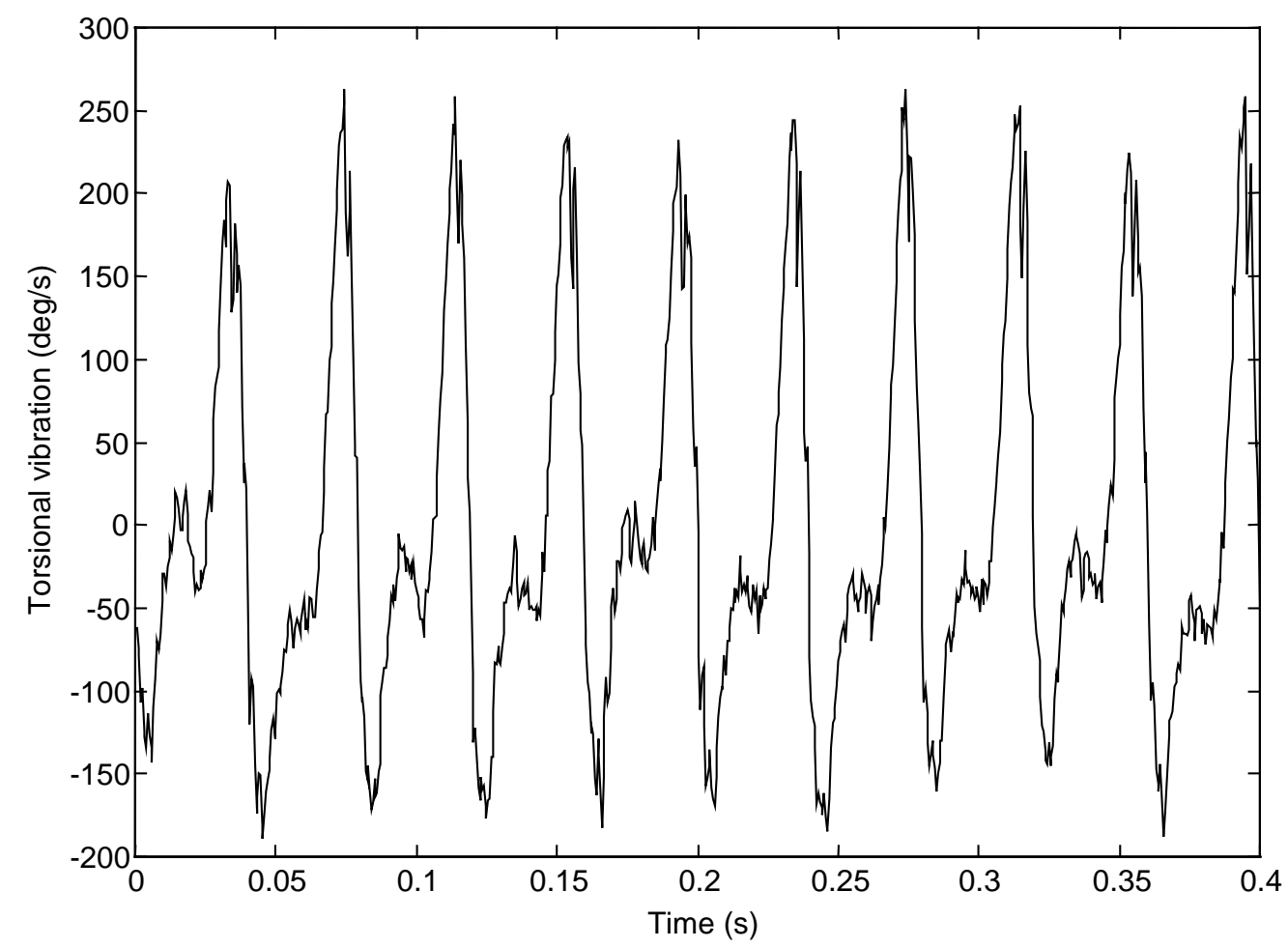

Figure 14

Torsional and Bending Vibration Measurement on Rotors using Laser Technology T.J. Miles, M. Lucas, N.A. Halliwell and S.J. Rothberg Loughborough University 


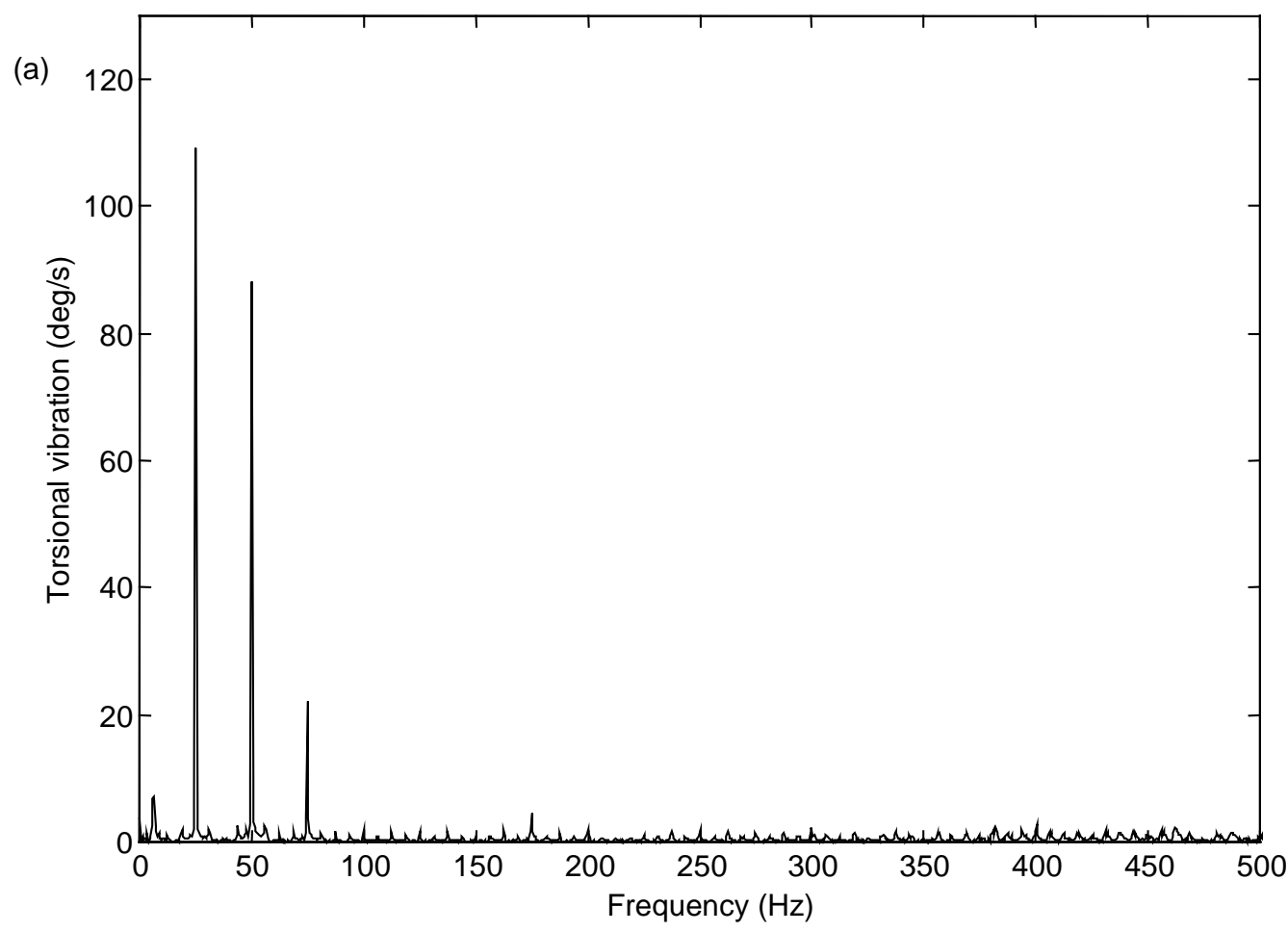

Figure 15a

Torsional and Bending Vibration Measurement on Rotors using Laser Technology

T.J. Miles, M. Lucas, N.A. Halliwell and S.J. Rothberg Loughborough University 


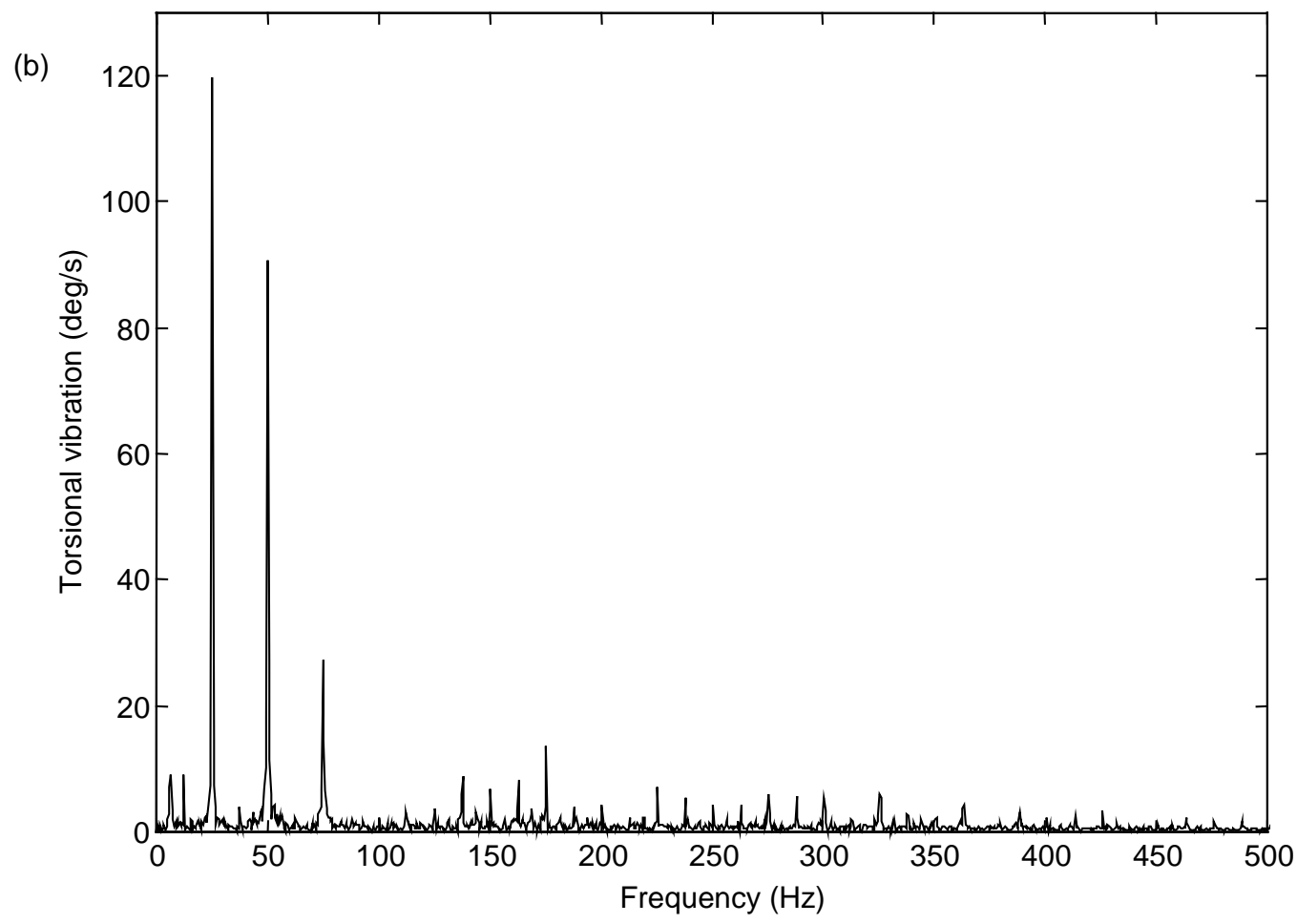

Figure 15b

Torsional and Bending Vibration Measurement on Rotors using Laser Technology

T.J. Miles, M. Lucas, N.A. Halliwell and S.J. Rothberg

Loughborough University 


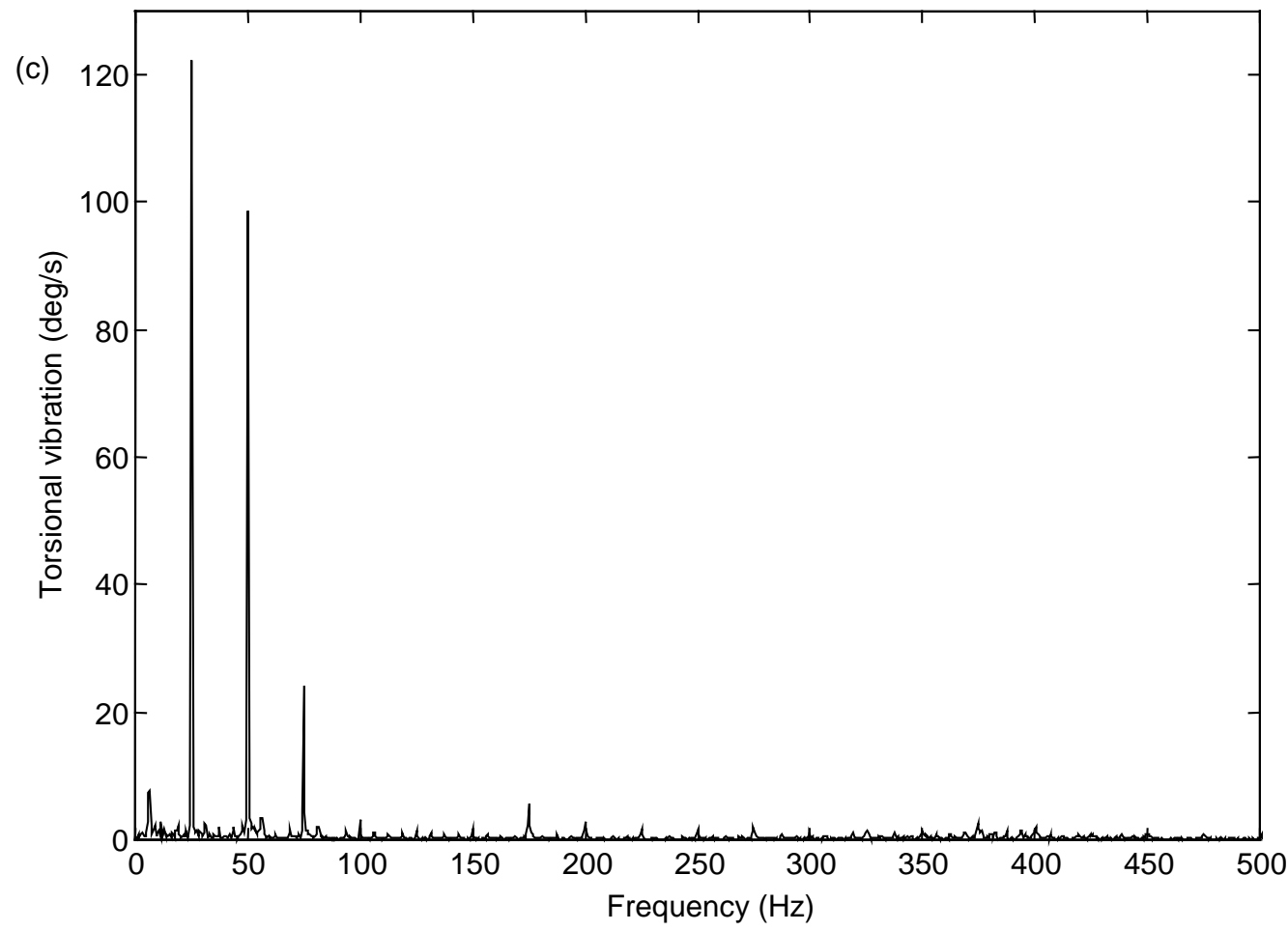

Figure 15c

Torsional and Bending Vibration Measurement on Rotors using Laser Technology

T.J. Miles, M. Lucas, N.A. Halliwell and S.J. Rothberg Loughborough University 


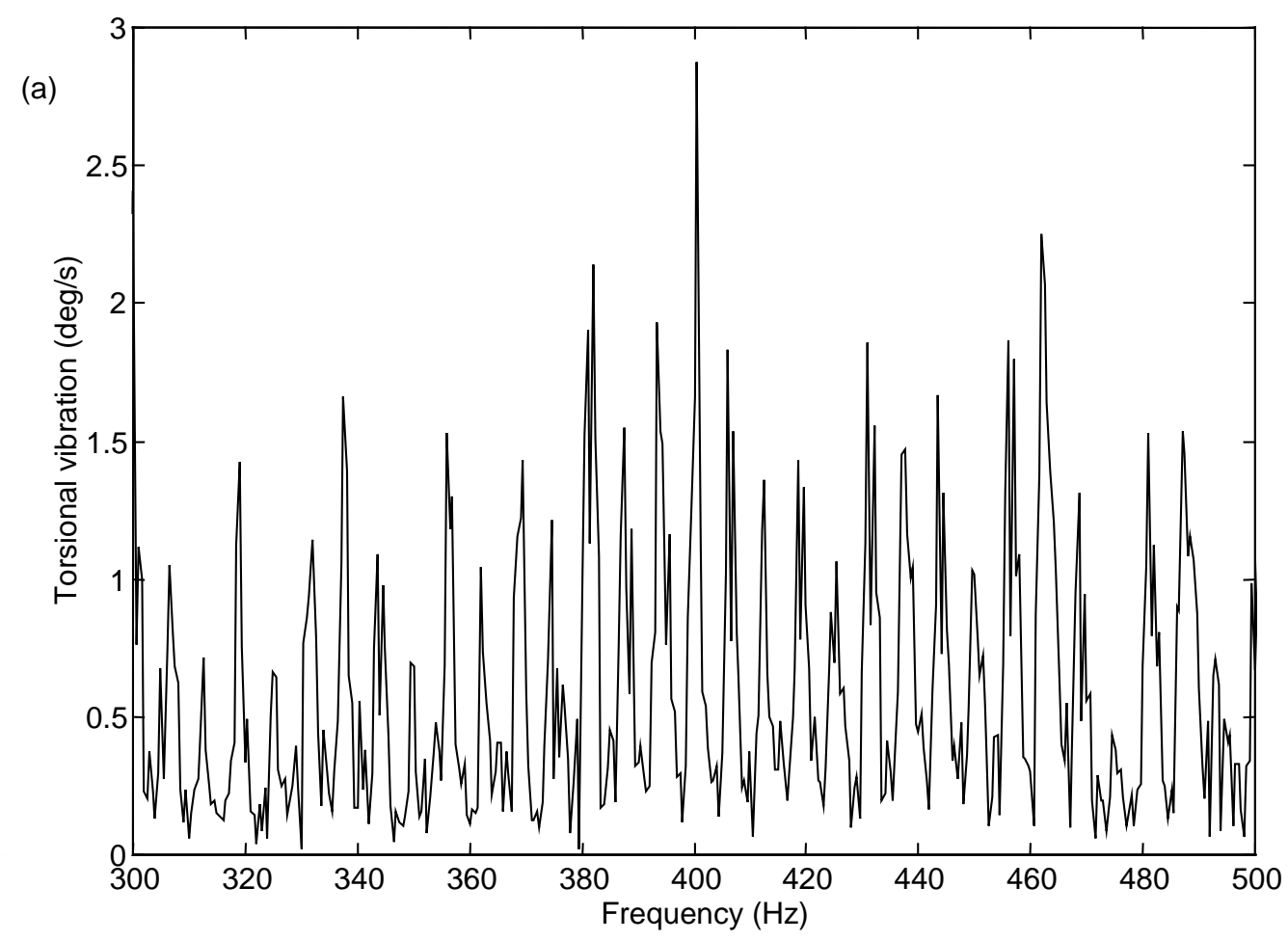

Figure 16a

Torsional and Bending Vibration Measurement on Rotors using Laser Technology

T.J. Miles, M. Lucas, N.A. Halliwell and S.J. Rothberg Loughborough University 


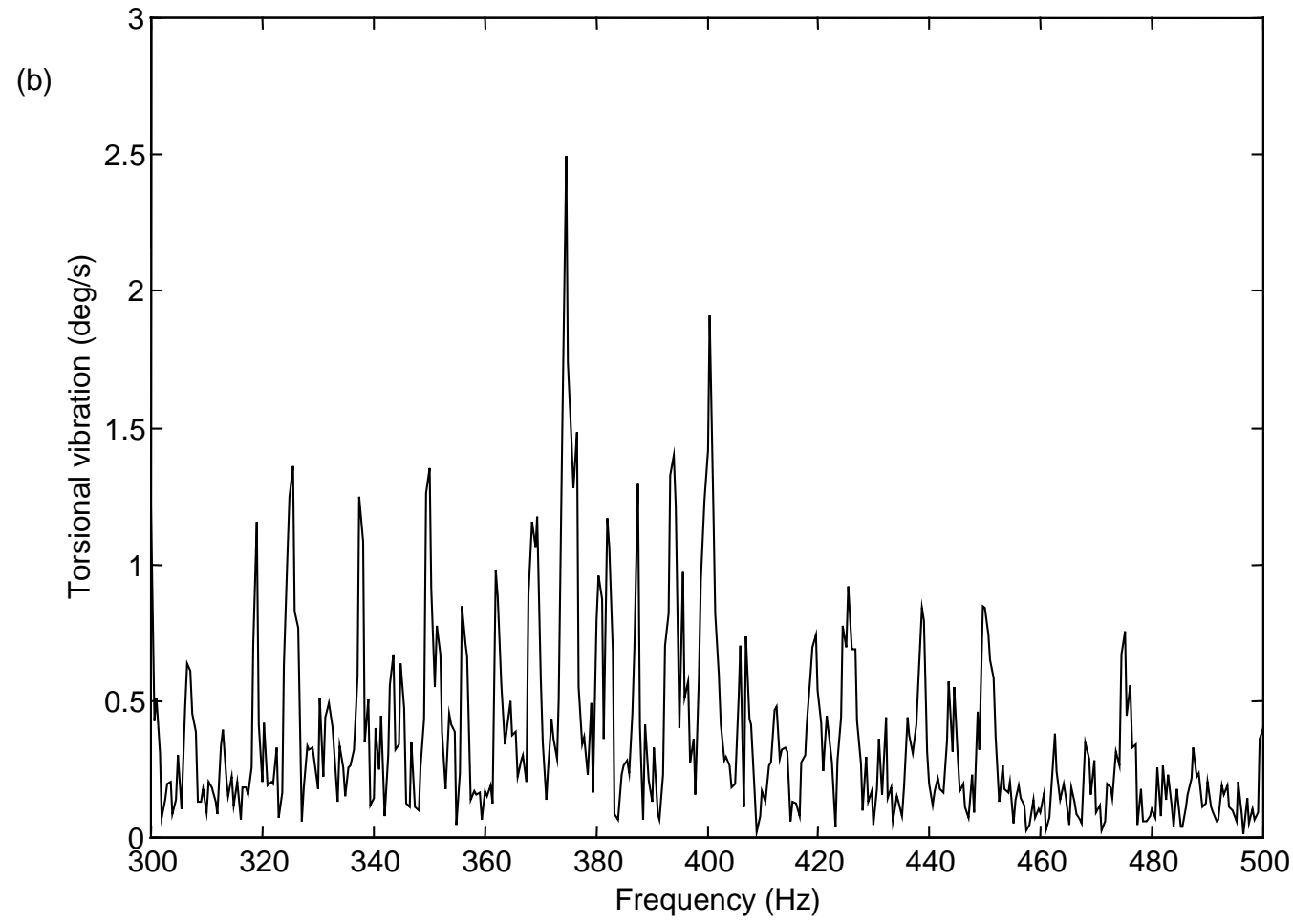

Figure 16b

Torsional and Bending Vibration Measurement on Rotors using Laser Technology

T.J. Miles, M. Lucas, N.A. Halliwell and S.J. Rothberg Loughborough University 


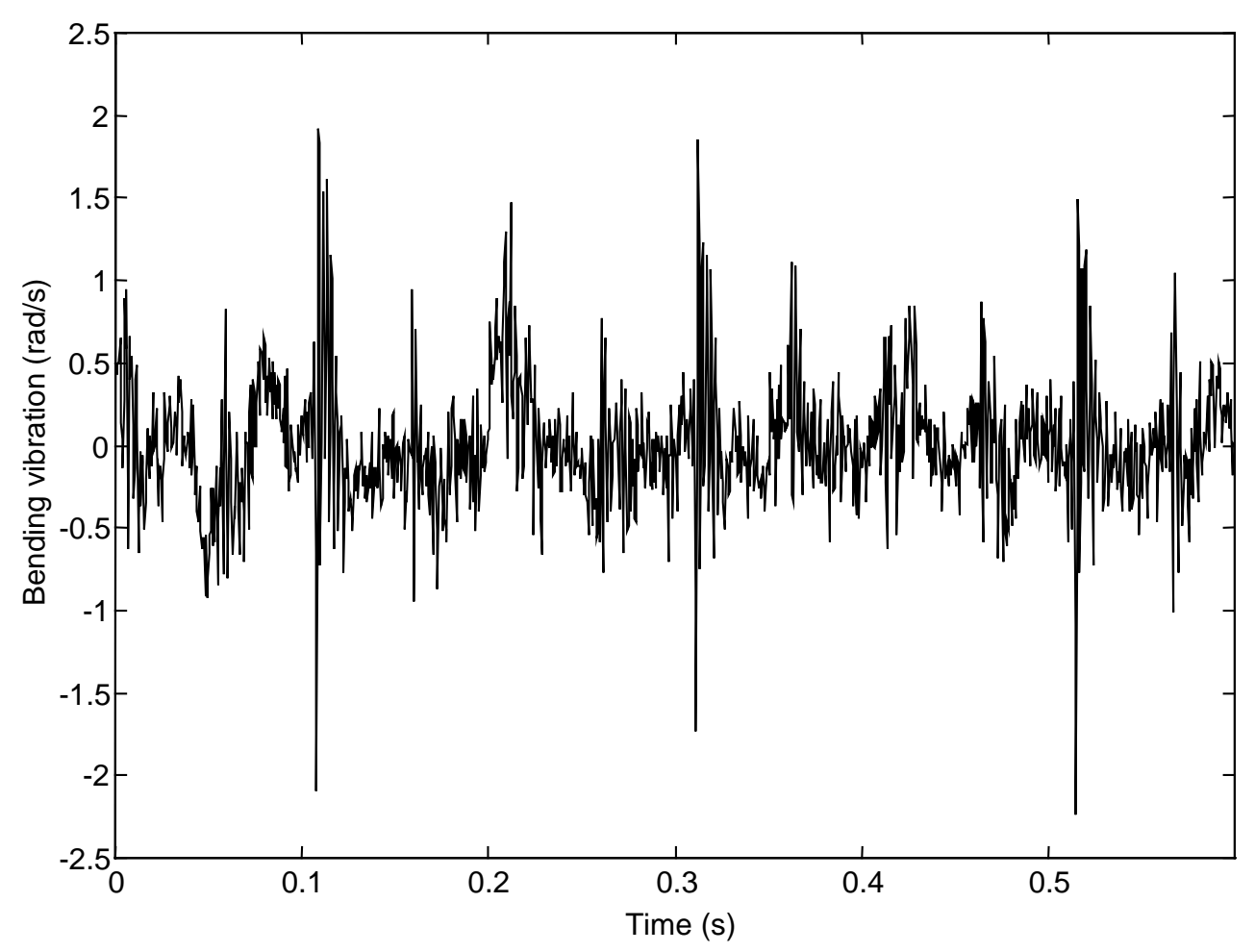

Figure 17

Torsional and Bending Vibration Measurement on Rotors using Laser Technology

T.J. Miles, M. Lucas, N.A. Halliwell and S.J. Rothberg Loughborough University 


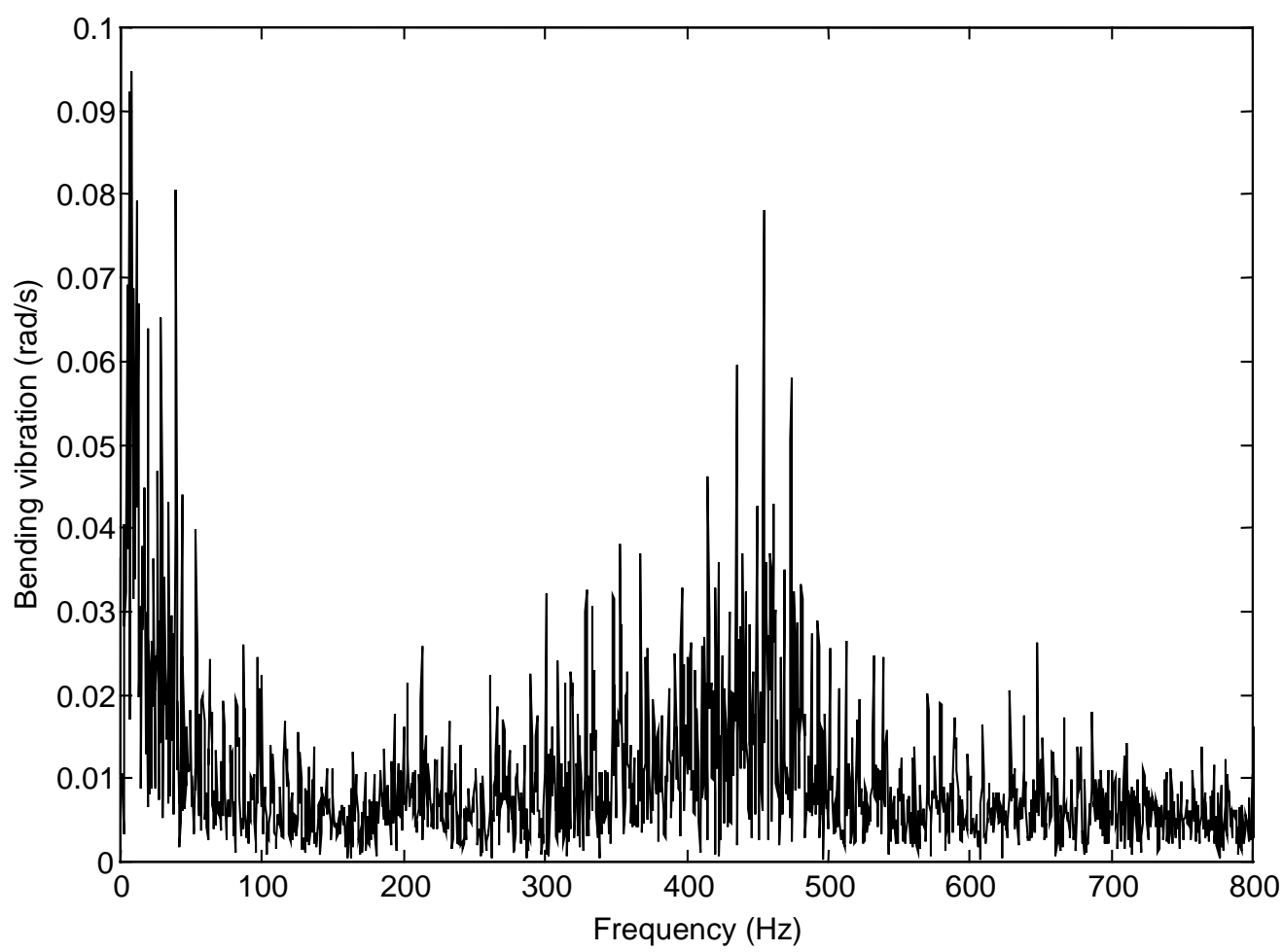

Figure 18

Torsional and Bending Vibration Measurement on Rotors using Laser Technology

T.J. Miles, M. Lucas, N.A. Halliwell and S.J. Rothberg Loughborough University 
Figure 19

Torsional and Bending Vibration Measurement on Rotors using Laser Technology

T.J. Miles, M. Lucas, N.A. Halliwell and S.J. Rothberg

Loughborough University 